\title{
ALGUNAS TÉCNICAS ESTAdísticas PARA ESTUDIAR EL CAMBIO EN LOS NIVELES DE CONCENTRACIÓN DE UNA VARIABLE*
}

\author{
WERNER ACKERMANN \\ $\mathbf{Y}$ \\ Fernando CoRtés \\ Flacso
}

\section{INTRODUCCIÓN}

LAS TÉCNICAS estadísticas que permiten estudiar la concentración ${ }^{1}$ de variables tales como el ingreso, la propiedad de la tierra, el acceso al crédito, etc.; han sido utilizadas con gran frecuencia. Entre ellas se ha recurrido con mayor insistencia al coeficiente de concentración de Gini y al diagrama de Lorenz. Sólo recientemente han surgido algunas aplicaciones del coeficiente entrópico de Theil."

En este trabajo se presenta una revisión somera de los aspectos que, a juicio de los autores, constituyen las propiedades básicas del índice de

* Una versión preliminar de este trabajo fue presentada en noviembre de 1978 en un seminario metodológico, organizado por el Prof. Ayrton Fausto en Quito (Ecuador) con el patrocinio conjunto de la Pontificia Universidad Católica del Ecuador y la Facultad Latinoamericana de Ciencias Sociales (Flacso), Sede Académica Quito.

1 Dado el tema central de este artículo es conveniente precisar desde la partida el uso que se dará al término concentración y establecer su vinculación con la idea de equidistribución.

No queremos que al encontrar este término el lector intuitivamente evoque un antónimo del concepto de dispersión, representándoselo entonces como la ubicación de las observaciones en el entorno de la media. Es decir, no debe pensarse en una relación inversa entre dispersión y concentración: a mayor dispersión, menor concentración y viceversa. Al contrario, cuando las observaciones se ubican en la vecindad de la media aritmética, diremos que hay menor concentración de la variable y cuando se alejan de la media, hablaremos de una mayor concentración.

Esta decisión se basa en el intento de establecer las vinculaciones entre los procedimientos estadísticos usuales para estudiar la concentración y la posible utilización de la noción de varianza para estos mismos fines. En el texto se muestra la equivalencia lógica entre la línea de equidistribución y la media aritmética.

2 Véase, por ejemplo, los trabajos del equipo de O. Altimir, de la CEPAL, y la investigación de W. Van Ginneken, "Análisis de descomposición del índice de Theil aplicado a la distribución del ingreso familiar en México", Economía y Demografía, Vol. IX, Núm. 1, 1975. 
Gini. Se tratará de establecer las condiciones que enmarcan su utilización corriente y desarrollar algunos caminos analíticos que emergen de su definición y que permitirían extender su aplicación.

Nuestro interés consiste básicamente en indagar respecto a técnicas estadísticas que permitan estudiar los cambios en los niveles de concentración. Es decir, no nos preocuparemos tanto por analizar maneras alternativas para medir el grado de concentración presente en una distribución de frecuencias, sino más bien se tiene interés por desarrollar algunas vías que permitan estudiar comparativamente la concentración de dos o más distribuciones.

El contraste entre los niveles de concentración exhibidos por dos o más distribuciones se puede referir a comporaciones ubicadas en el tiempo o en el espacio. En el primer caso se trataría de indagar, por ejemplo, la tendencia que ha seguido el cambio en la distribución del ingreso o de la propiedad agrícola a lo largo de varios años. En el segundo, se analizarían las diferencias entre las distribuciones de ingreso o de propiedad en varias regiones de un mismo país o entre varios países. A partir de la delimitación de la problemática que se pretende abordar en este artículo, definida dentro del ámbito técnico-estadístico y centrada en el análisis de la variación experimentada por los grados de concentración de dos o más distribuciones de frecuencias, se propone como tarea examinar las potencialidades del índice de concentración de Gini e investigar la pertinencia de la utilización del concepto de varianza para estos fines.

De acuerdo a estas orientaciones generales, se organizan los temas a desarrollar de la siguiente manera:

1). Revisión de las propiedades básicas del coeficiente de concentración de Gini, con sugerencias de algunas vías para extender su campo de aplicación (segunda sección).

2). Desarrollo del teorema de la descomposición de la varianza para su uso en el análisis de las alteraciones en los niveles de concentración de dos o más distribuciones (tercera sección).

3). Una aplicación de la técnica expuesta en la tercera sección a un conjunto de datos reales (cuarta sección).

Dado nuestro interés -en este trabajo- por los aspectos técnicos y metodológicos involucrados en este tipo de estudio, nos limitaremos a destacar las potencialidades analíticas del instrumento, obviando consideraciones de orden teórico y práctico que podrían surgir con facilidad del examen de los datos.

4). En la última sección se presentan las conclusiones generales que se derivan del material expuesto a lo largo del trabajo.

\section{ORIGEN DEL PROBLEMA}

El índice de Gini ha sido utilizado de manera habitual como un indicador del grado de concentración existente en la distribución de una va- 
riable. Intenta reflejar cómo se distribuye un monto total entre un conjunto de unidades. Así, por ejemplo, puede utilizarse para medir el grado de concentración del volumen total de créditos existentes en un país (para un periodo determinado) entre el conjunto de establecimientos industriales, o bien para analizar el grado de concentración de la propiedad de la tierra entre distintos tipos de tenencias, o la distribución más o menos equitativa del capital manufacturero entre los empresarios, etc.

El coeficiente de Gini se usa con particular frecuencia para el estudio de la concentración del ingreso. En este tipo de aplicación se busca disponer de una medida global que permita caracterizar la forma como se reparte el producto de la actividad económica entre distintas unidades sociales. Aun cuando lo habitual sea utilizar tramos de ingresos como unidades (se analiza la distribución del ingreso entre categorías estadísticas), nada impediría en principio ${ }^{3}$ que ellas estén formadas por agregados de otra naturaleza, tales como categorías ocupacionales o clases sociales.

Si bien el estudio del nivel de concentración del ingreso puede tener un interés teórico y práctico en sí, no son escasos los análisis donde el objetivo reside en la comparación entre dos o más momentos en el tiempo para una misma distribución, o entre dos o más puntos en el espacio, para distintas distribuciones. En el primer caso, el propósito podría consistir en examinar cómo la política global de desarrollo aplicada sobre cierto lapso ha incidido en el proceso de concentración o desconcentración del ingreso. En el segundo caso, lo que podría interesar sería contrastar estilos o modelos de desarrollo que conducen a diferentes maneras de repartir el producto social.

Sin embargo, cabe señalar que al comparar los grados de concentración entre dos o más distribuciones (ya sea en el espacio o en el tiempo) debe distinguirse con nitidez entre los aspectos relativos y absolutos involucrados en la comparación. En efecto, se podría observar parà un mismo país el aumento del nivel de concentración del ingreso en dos momentos en el tiempo, sin que ello implique un deterioro absoluto de la capacidad de compra de los consumidores, por cuanto podría haber ocurrido simultáneamente un aumento sustancial en los valores medios de los ingresos reales en todas y cada una de las distintas unidades. Es decir, si bien el ingreso se ha concentrado ello se ha producido conjuntamente con una elevación de los ingresos medios.

Atendamos, en primer lugar, a los aspectos relativos contenidos en la idea de concentración. El estudio de la distribución de una variable entre un conjunto de unidades, implica de manera necesaria una comparación entre la distribución observada y una distribución teórica. Es decir, la mayor o menor concentración se definirá con respecto a una distribución teórica de frecuencias, construida sobre la base de una norma. La idea nor-

3 Para su cálculo el índice de Gini supone que las observaciones deben ordenarse. La jerarquización de los datos se pueden hacer fácilmente al interior de una categoría. Pero es posible que surjan dificultades en el ordenamiento de las características cuando se superponen las observaciones. 
mativa que subyace al coeficiente de concentración de Gini es la de una distribución "democrática": ${ }^{4}$ si las unidades observadas son individuos, a cada quien le corresponderá lo mismo; si son agregados, a cada uno corresponderá un monto proporcional a su tamaño. Esta norma se expresa geométricamente por medio de la línea de equidistribución; también podría representarse aritméticamente a través de un valor medio: si a cada unidad individual le corresponde un valor igual al de la media se tendría un caso de equidistribución perfecta; lo mismo acontecería si cada agregado obtuviese un monto igual al producto de su tamaño por el valor medio.

En cuanto a los aspectos absolutos involucrados en la comparación de grados de concentración entre distribuciones, hay que notar que las normas a las que se refieren los aspectos relativos, pueden estar situadas en distintos niveles. Así, por ejemplo, si la norma democrática es expresada a través de la media aritmética, dos distribuciones pueden tener diferentes valores medios aunque presenten el mismo grado de concentración. El caso inverso sería el de dos o más distribuciones de frecuencias que presenten el mismo valor medio, pero diferente grado de concentración. ${ }^{5}$ En cualquier situación real con seguridad nos encontramos con mezclas en que ha variado tanto la concentración como el nivel absoluto de ingreso. En la sección siguiente se presenta un desarrollo que permitiría separar entre ambas fuentes de variación.

De las líneas anteriores puede extraerse como conclusión parcial que en el uso del coeficiente de Gini con fines comparativos, deben tomarse precauciones para no inferir a partir de una diferencia entre dos valores un mejoramiento o un deterioro de los montos absolutos de ingresos percibidos por las unidades. Esta reserva metodológica no emerge de una deficiencia inherente al indicador de Gini, sino que surge debido al intento de emplearlo para predicar sobre cambios en la distribución. El coeficiente ha sido diseñado para medir concentración en una distribución. Su aplicación sobre varias distribuciones de frecuencias, para analizar sus diferencias en cuanto a concentración, implica una extensión en el empleo del índice que fuerza los límites de su construcción. Es por ello que su uso comparativo debe ser acompañado de las precauciones que se han indicado.

Además de esta limitación en cuanto a la aplicación del coeficiente de Gini, es necesario tener en cuenta un problema específico que surge también en el ámbito de la comparación, pero que puede ser considerado como una debilidad en su construcción.

Como es sabido, el índice de concentración de Gini se define como la

4 Véase H. Alker, El uso de la matemática en el análisis político, Buenos Aires, Amorrortu Editores, 1969, Cap. III.

5 Se podría agregar una tercera dimensión en el diagrama de Lorenz que representaría los valores medios. A partir de este diagrama sería posible definir un coeficiente de Gini en el espacio, que tomaría en cuenta tanto los aspectos absolutos como relativos involucrados en la concentración. 
relación entre el área limitada por la línea de equidistribución y la curva de Lorenz, y el área del triángulo rectángulo cuya hipotenusa es la línea de equidistribución y los catetos son las frecuencias relativas acumuladas de las unidades y de la variable.

El coeficiente se puede definir gráficamente como el cociente entre el área $n$ y el área del triángulo rectángulo $O A B$, del diagrama de Lorenz (véase la gráfica 1 ).

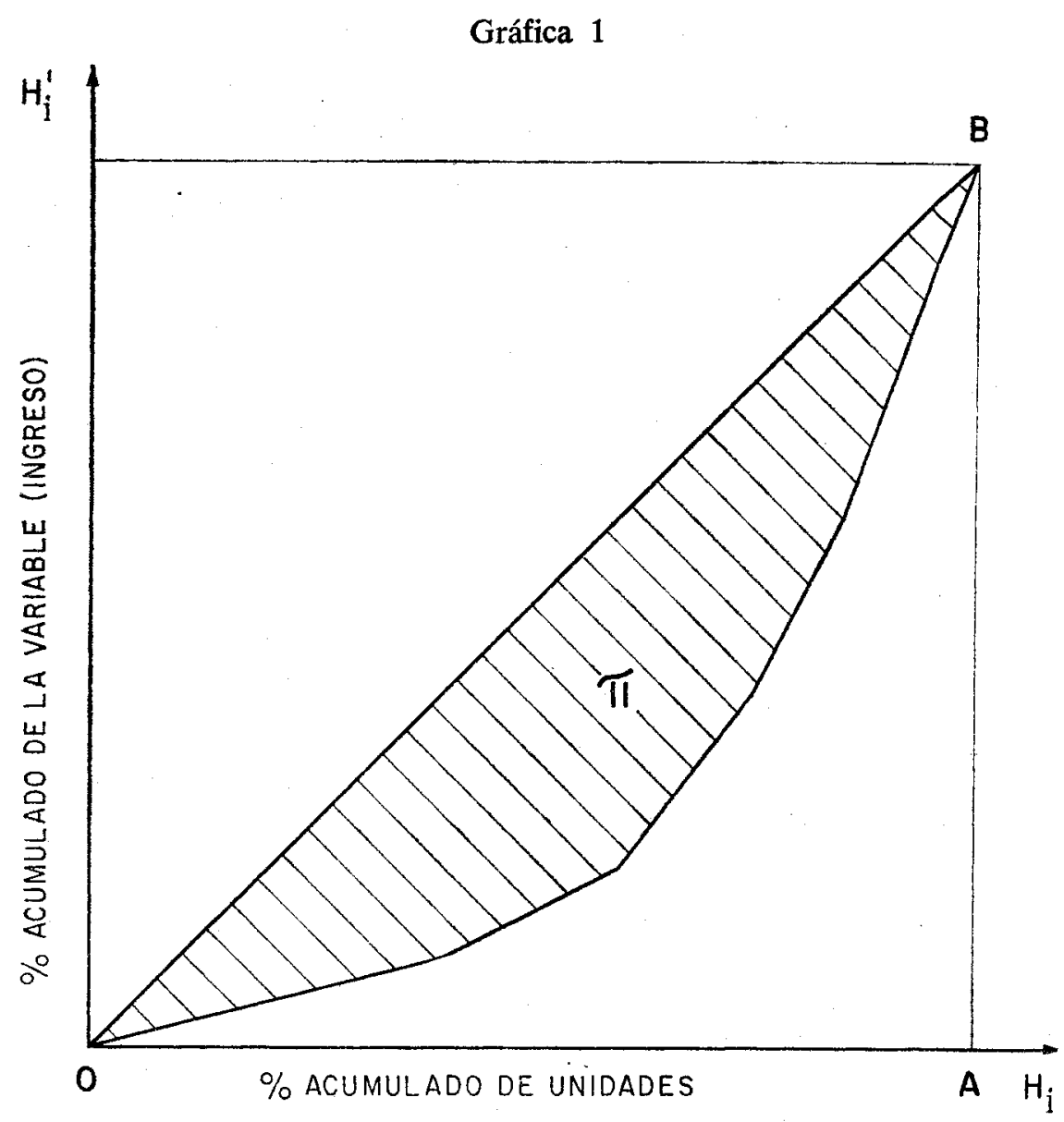

De la gráfica se desprende que el área $\pi$ sólo cambiará de valor en función de alteraciones en la forma de la curva de Lorenz, por cuanto la línea de equidistribución es constante por representar la norma. Esto quiere decir que en el diagrama de Lorenz el área del triángulo $O A B$ será -constante (e igual a 0.5) cualquiera que sea la curva de Lorenz. En cambio no acontece lo mismo con el área de $\pi$, puesto que su valor depende 


\section{Gráfica 2}

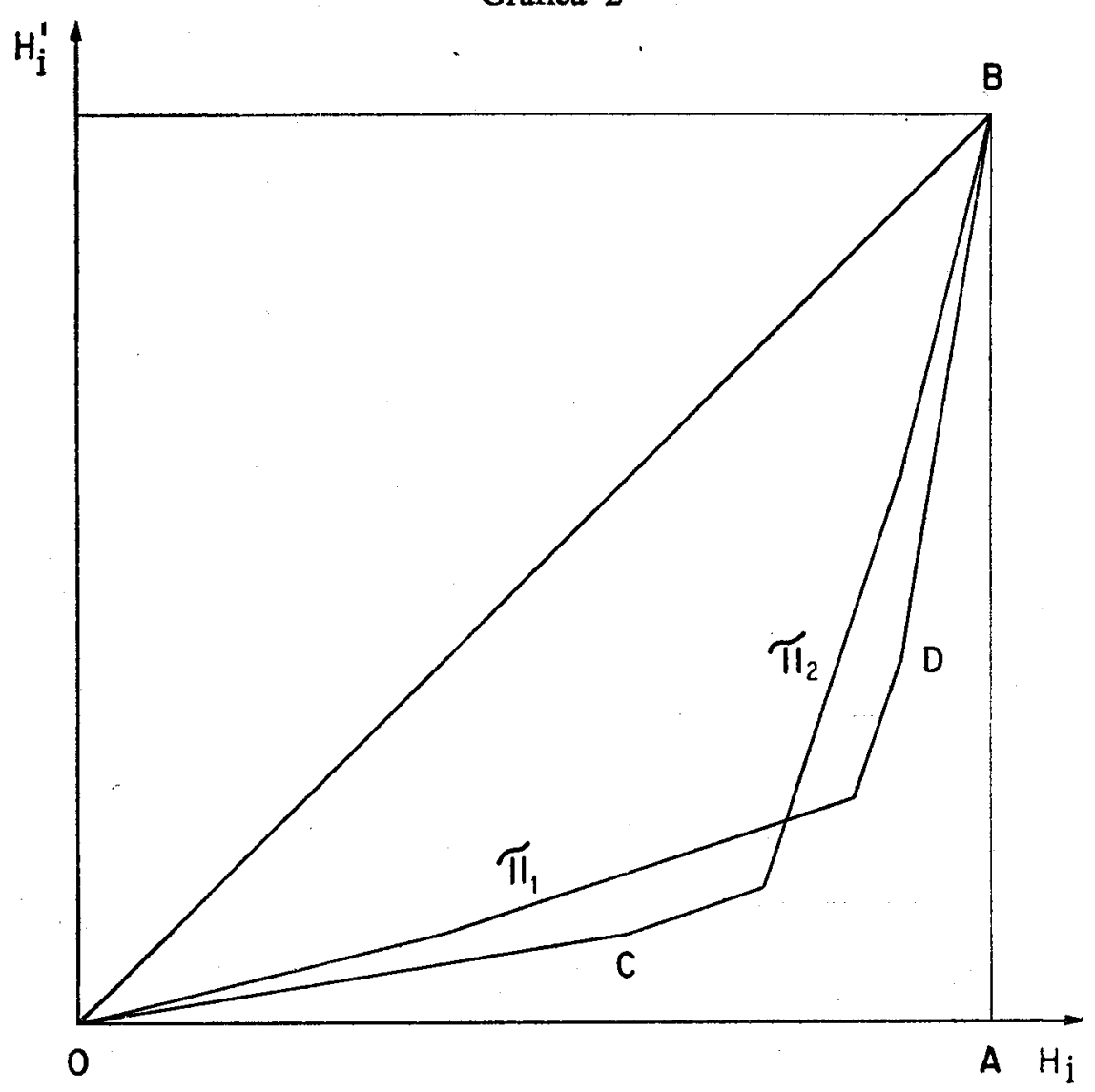

de la cercanía o lejanía de dicha curva en relación a la equidistribución. Ahora bien, el grado de aproximación entre ambas líneas puede ser interpretado como una característica agregada de la distribución de frecuenciąs, ya que dos o más distribuciones pueden presentar el mismo grado de aproximación, pero diferir en su forma. Para ilustrar este caso se incluye la gráfica 2 , en la que se representan dos curvas de Lorenz (las curvas $O C B$ y $O D B$ ) que tienen distinta forma, pero que presentan el mismo grado de aproximación a la recta de equidistribución, lo que conduce a que ambas áreas sean iguales $\left(\pi_{1}=\pi_{2}\right)$ así como a un mismo valor del coeficiente de concentración de Gini.

Si bien el índice de Gini puede ser considerado como un buen indicador del nivel agregado de concentración de una variable, presenta la limitación de no ser sensible a la manera como se llega a ese resultado. Así por ejemplo, en el estudio comparativo de dos distribuciones de ingreso se podría encontrar un mismo nivel agregado de concentración, a pesar 
de que en un caso el ingreso puede estar concentrado en los tramos altos y en el otro en los estratos medios. Tanto desde el punto de vista teórico como práctico, las dos situaciones descritas suelen conducir a conclusiones de distinta naturaleza, las cuales pasarían inadvertidas si sólo se considerase exclusivamente el valor del coeficiente de Gini.

Para ilustrar cuantitativamente estas ideas se consideran las distribuciones hipotéticas de frecuencias de la variable ingreso personal que aparecen en los cuadros 1 y 2 .

\section{Cuadro 1}

DistRIBUCIÓN DE FRECUENCIAS I

\begin{tabular}{rccc}
\hline Niveles & Ingresos & $\begin{array}{c}\text { Perceptores } \\
(\mathrm{g})\end{array}$ & $\begin{array}{c}\text { Inoreso } \\
(8)\end{array}$ \\
\hline I & $0-10$ & 0.35 & 0.15 \\
II & $10-20$ & 0.30 & 0.20 \\
IV & $20-30$ & 0.20 & 0.25 \\
V & $30-40$ & 0.10 & 0.30 \\
Total & $40-50$ & 0.05 & 0.10 \\
\hline
\end{tabular}

\section{Cuadro 2}

DISTRIBUCIÓN DE FRECUENCIAS II

\begin{tabular}{rccc}
\hline Niveles & Ingresos & $\begin{array}{c}\text { Perceptores } \\
(8)\end{array}$ & $\begin{array}{c}\text { Ingreso } \\
(8)\end{array}$ \\
\hline I & $0-10$ & 0.35 & 0.100 \\
II & $10-20$ & 0.30 & 0.300 \\
III & $20-30$ & 0.20 & 0.225 \\
IV & $30=40$ & 0.10 & 0.275 \\
V & $40-50$ & 0.05 & 0.100 \\
Tota1 & & 1.00 & 1.000 \\
\hline
\end{tabular}

Obsérvese que ambos cuadros tienen en común los intervalos de clases (tramos de ingresos) y las frecuencias relativas de las unidades (proporción de la población en cada tramo de ingreso). Por lo tanto, ambas distribuciones tienen la misma media, vale decir, el nivel de la norma es idéntico. ${ }^{6}$

Como el valor del coeficiente de concentración es el mismo para ambas distribuciones $(0.3625)$ y habiendo tomado la precaución de considerar el nivel absoluto (que en este caso es constante) se podría concluir de manera apresurada que ambas distribuciones presentan la misma "concentración".

Sin embargo, se observa que la forma en que se produce la concentra-

- Se supone que la media se calcula a través de la ponderación de las marcas de clases por las frecuencias relativas de los perceptores. 
ción es sensiblemente diferente. En efecto, en el primer cuadro la distribución del ingreso se concentra modalmente en el cuarto tramo; en tanto que en la segunda se producen concentraciones modales en el segundo y en el cuarto intervalo.

Si estos cuadros correspondiesen a las distribuciones de ingreso de un país en dos momentos sucesivos en el tiempo, expresarían cambios de importancia inducidos tal vez por la política económica. De una situación que se caracterizaba por concentrar el poder comprador en el tramo medio alto (cuarto intervalo) se ha pasado a otra en que se aglutina en el segundo y cuarto tramos. Estas alteraciones con toda seguridad se habrán traducido en modificaciones del perfil de la demanda, sin cambios en el nivel absoluto del ingreso ni tampoco en el grado global de concentración.

De manera lamentable, los casos concretos mezclan los tres órdenes de elementos que intervienen en los cambios de los niveles de concentración: a) cercanía o lejanía con respecto á la norma, es decir, el grado de concentración; $b$ ) la forma de la curva de Lorenz (forma de la concentración); y c) el nivel de la norma. Esto quiere decir que el análisis concreto supone desentrañar el papel que le corresponde a cada uno de estos elementos. Con el propósito de acercarnos a las condiciones reales del análisis estudiemos la alteración en la "concentración" del ingreso en áreas urbanas que se produjo en Ecuador entre los años 1968 y 1975 (véase el cuadro $3)$.

\section{Cuadro 3}

ECUAdor: Población ocupada POR ESTRatos DE INGRESO (ÁREA URBANA), 1968 y 1975

(Datos en millones de sucres a precios de 1972)

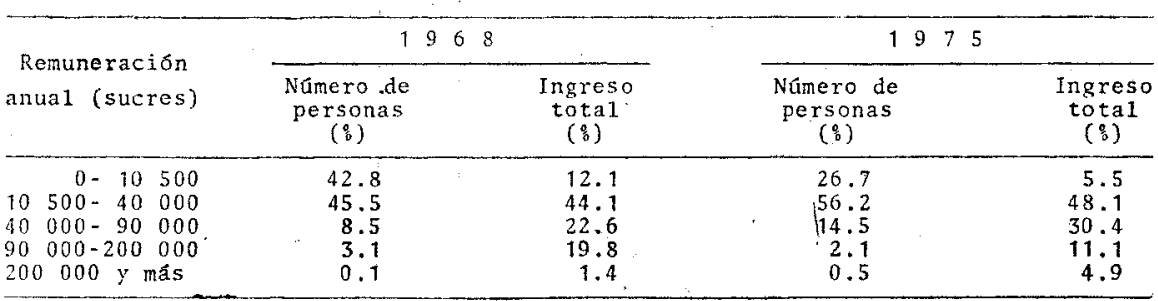

Fuente: Junta Nacional de Planificación, INEC-Encuesta de Hogares, 1968 y de Población y Ocupación, 1975 (Área urbana).

Del cuadro 3 se deriva: a) Un leve proceso de desconcentración del ingreso. El coeficiente de Gini disminuye desde $\mathrm{G}=0.465$ a $\mathrm{G}=0.406$. b) Las alteraciones más importantes que conducen a la desconcentración del ingreso serían una disminución significativa de perceptores en el tramo más bajo, acompañada por un aumento de importancia en los tamaños relativos de los tramos 2 y 3 (que podríamos caracterizar como medio bajo 
y medio). Esta nueva situación se aproxima a lo que Jorge Graciarena ${ }^{7}$ ha denominado proceso de redistribución mesocrática, en oposición a la concentración "elitaria" que se define por una redistribución en favor de los tramos más altos. c) También hay que observar el aumento experimentado por el ingreso medio anual, el cual se ha elevado en este lapso en un $22.7 \%$.

Estos cambios en la distribución del ingreso, que en última instancia se manifiestan en modificaciones en el perfil de la demanda, parecen ser coherentes con el camino seguido en materia de industrialización por Ecuador. ${ }^{8}$

En síntesis, el coeficiente de Gini parece ser un buen instrumento para indicar el grado de concentración global de una distribución.

Cuando se trata de estudiar comparativamente los niveles de concentra-

\section{Gráfica 3}

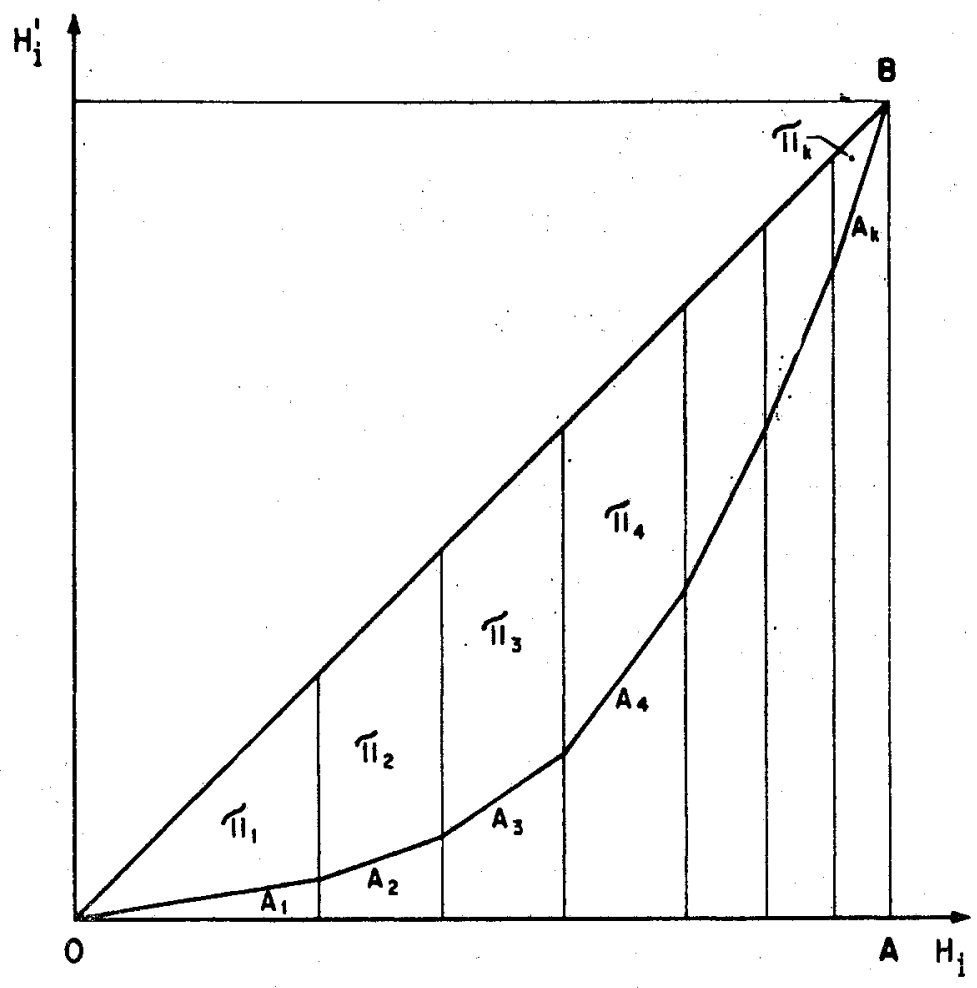

7 J. Graciarena, "Tipos de concentración del ingreso y estilos políticos en América Latina", en Revista de la CEPAL, 2o. semestre de 1976, pp. 203-237.

8 Para mayores detalles, consúltese J. Moncada y F. Villalobos, "Distribución del ingreso, estructura productiva y alternativas de desarrollo", Mimeo, FLACso-Quito, 1978. 
ción de dos o más distribuciones (diferenciadas en el tiempo o en el espacio) interesa no sólo tener una indicación relativa de la concentración de la distribución como un todo, sino también disponer de criterios que permitan juzgar acerca de los cambios absolutos en los niveles de la norma $\mathrm{y}$ de las diferencias en las formas de las curvas de Lorenz.

El primero de estos aspectos puede detectarse a través de una comparación de los valores medios globales, mientras que para el segundo habría por lo menos dos caminos abiertos. Uno consistiría en la simple extensión de la idea básica que define al coeficiente de Gini (la relación entre el área formada por la línea de equidistribución y la curva de Lorenz, y el área del triángulo rectángulo) pero esta vez aplicado sobre cada intervalo de clase. Se establecería entonces, para cada tramo, la relación entre el área delimitada por los segmentos de la línea de equidistribución y de la curva de Lorenz correspondientes a este intervalo y el área total definida por el mismo segmento de la línea de equidistribución y el intervalo de clase.

Estas ideas se pueden expresar como se muestra en la gráfica 3.

Para analizar la forma de la distribución global bastaría con dividir los valores correspondientes a las áreas $\pi_{i}\left(\pi_{1}, \pi_{2}, \ldots \pi_{k}\right)$ por las correspondientes áreas totales para cada tramo de la curva de Lorenz $A_{i}\left(A_{1}, A_{2}, \ldots A_{k}\right)$. De esta manera se calcularian $k$ coeficientes Giniintervalos que podrían ser usados como indicadores de la forma de concentración. De este modo se habría definido:

$$
G_{i}=\frac{\pi_{i}}{A_{i}} \quad(i=1,2,3, \ldots, k)
$$

Para obtener el coeficiente global de Gini bastaría con ponderar cada uno de los coeficientes Gini-intervalo por la relación entre el área total correspondiente al segmento de la curva de Lorenz y el área de triángulo $O A B$. Con las ponderaciones $A_{i} / 1 / 2$ se concluye que:

$$
\begin{aligned}
& G^{*}=2 \sum_{i=1}^{k} G_{i} A_{i}=2\left[\frac{\pi_{1}}{A_{1}} A_{1}+\frac{\pi_{2}}{A_{2}} A_{2}+\ldots+\frac{\pi_{k}}{A_{k}} A_{k}\right] \\
& G^{*}=\frac{\sum_{i=1}^{k} \pi_{i}}{1 / 2}=\frac{\pi}{1 / 2} ; \text { ya que } \sum_{i=1}^{k} \pi_{i}=\pi
\end{aligned}
$$

Luego $G^{*}=G$.

Es decir, la suma ponderada de los coeficientes Gini-intervalo iguala al valor global del coeficiente.

Sin embargo, en este trabajo no se desarrola esta línea pero se presenta en la siguiente sección un camino alternativo, particularmente apto para detectar cambios en los niveles y formas de la concentración. 


\section{UN :MÉTODO PARA ESTUDIAR VARIACIONES DE LA CONCENTRACIÓN}

En la sección anterior se ha visto que la norma "democrática" con respecto a la cual se mide el grado de concentración de una distribución de frecuencias puede ser expresada geométricamente por la línea de equidistribución y aritméticamente a través de la media.

Las desviaciones de los valores de variable con respecto a la media aritmética pueden ser interpretadas como una medida del grado de aproximación o lejanía en relación a la norma "democrática". Ahora bien, la varianza se puede definir conceptualmente como la media del cuadrado de las desviaciones con respecto a la media aritmética y: por lo tanto puede ser utilizada como un indicador de la concentración de la distribución.

En realidad la varianza mantiene una relación directa con la dispersión de una distribución, es decir, con el grado de alejamiento de las observaciones con respecto a la media. En consecuencia, la vinculación con la concentración es inmediata: a mayor varianza mayor grado de concentración y viceversa. ${ }^{9}$

Por otro lado, habría que señalar que la varianza difícilmente puede ser utilizada como un índice del grado de concentración de una distribución, por cuanto no tiene un límite superior conocido, además de que su valor es afectado por cambios de escala.

Una propiedad muy interesante de la varianza consiste en la posibilidad de descomponerla en inter e intravarianza. Si se divide un conjunto de observaciones en $G$ grupos, se pueden realizar dos tipos de cálculos de varianzas: uno que tiene en cuenta la dispersión de las medias de los $G$ grupos con respecto a la media general de la distribución y que origina la intervarianza y otro que considera las dispersiones al interior de cada uno de los $G$ grupos, cuya media genera la intravarianza de la distribución. Es conocido el teorema que establece que el valor de la varianza total es igual a la suma de la inter más la intravarianza. Es decir:

$$
S^{2}=S_{d}^{2}+S_{e}^{2}
$$

donde $S^{2}$ simboliza la varianza total de la distribución, $S_{d}^{2}$ denota la intravarianza y $S_{e}^{2}$ representa la intervarianza. Las fórmulas de cálculo son:

$$
S^{2}=\Sigma\left(Y_{i}-\bar{Y}\right)^{2} h_{i}
$$

en donde $Y_{i}$ simboliza los distintos valores de variable y $\bar{Y}$ la media general de la distribución y $h_{i}$ las frecuencias relativas de cada intervalo de clase.

9 Si $G=0$, la varianża también será nula, ya que el área entre las líneas de equidistribución y de Lorenz sería igual a 0 debido a que a cada unidad le ha correspondido un valor igual a la media general. 


$$
S_{d, g}^{2}=\sum_{i=1}^{G}\left(Y_{g i}-Y_{g}\right)^{2} h_{g, i} \quad(g=1,2, \ldots G)
$$

Esta fórmula corresponde al cálculo de la varianza dentro del grupo $g$, donde $Y_{g, i}$ denota los distintos valores de variable al interior de $g, \bar{Y}_{g}$ la media del grupo $g, h_{g, i}$ las frecuencias relativas correspondientes a los intervalos de clases en el interior de $g$, y $n_{g}$ el número de observaciones en $g$.

$$
S_{d}^{2}=\sum_{g=1}^{G} S_{d, g}^{2} h_{g}
$$

La ecuación 4 representa la intravarianza de la distribución, donde $h_{g}$ simboliza la importancia relativa del grupo $g$ dentro del total; por lo tanto:

$$
h_{g}=n_{g} / n
$$

donde $n$ es el total de las observaciones de la distribución.

Por último,

$$
S_{e}^{2}=\sum_{g=1}^{G}\left(\bar{Y}_{g}-\bar{Y}\right)^{2} h_{g}
$$

es la fórmula intervarianza. Todos los términos que componen esta expresión han sido previamente definidos.

Como el interés radica en el estudio de las variaciones en la concentración ya sea a través del tiempo o del espacio, es necesario referir las distintas varianzas a un punto específico. En la próxima sección se incluye una aplicación de los procedimientos aquí desarrollados para descomponer el cambio de la concentración del ingreso a través del tiempo; por ello se presentan estos desarrollos referidos al tiempo. Esto no quiere decir que el procedimiento aquí expuesto sólo sea útil para hacer comparaciones temporales, ya que nada - desde el punto de vista técnico- impide hacerlo en el espacio.

Simbolicemos por $S_{p}^{2}$ a la varianza total de la distribución en el tiempo $p, y$ por $S_{p+r}^{2}$ a la misma varianza, pero esta vez referida al tiempo $p+r$.

Sean $S_{d, p}^{2}$ y $S_{d, p+r}^{2}$ las intravarianzas en los momentos $p$ y $p+r$, respectivamente.

Representemos por $S_{e, p}^{2}$ y $S_{e, p+r}^{2}$ las intervarianzas en los instantes $p$ y $p+r$.

De acuerdo con el teorema de la descomposición de la varianza tendremos: 


$$
\begin{aligned}
& S_{p}^{2}=S_{d, p}^{2}+S_{e, p}^{2} \\
& S_{p+r}^{2}=S_{d, p+r}^{2}+S_{e, p+r}^{2}
\end{aligned}
$$

La diferencia entre (7) y (8) da como resultado:

$$
S_{p}^{2}-S_{p+r}^{2}=\left(S_{d, p}^{2}-S_{d, p+r}^{2}\right)+\left(S_{e, p}^{2}-S_{e, p+r}^{2}\right)
$$

Esta ecuación permite descomponer el cambio que ha experimentado la concentración de la variable entre los instantes $p$ y $p+r$ en dos componentes: la parte del cambio total que es debida a la mayor o menor concentración en el interior de cada grupo y aquélla debida a la mayor o menor concentración entre los $G$ grupos. Es decir, el teorema de la descomposición de la varianza ayuda a identificar las fuentes que determinan el cambio en el nivel de concentración de las dos distribuciones. Si por ejemplo los $G$ grupos representan un conjunto de clases sociales y entre los dos periodos de tiempo ha habido una alteración en las pautas de la distribución del ingreso, se puede individualizar la parte que se debe al cambio producido en el interior de las clases (intravarianzas) y aquélla que se modifica como consecuencia de un acercamiento o alejamiento de las medias de las $G$ clases con respecto a la media general (intervarianza).

Ambos movimientos pueden captarse con mayor nitidez en la gráfica 4.

Entre las curvas $p$ y $p+r$ se advierte un cambio en la posición y en la dispersión. La distribución $p+r$, además de mostrar una media más

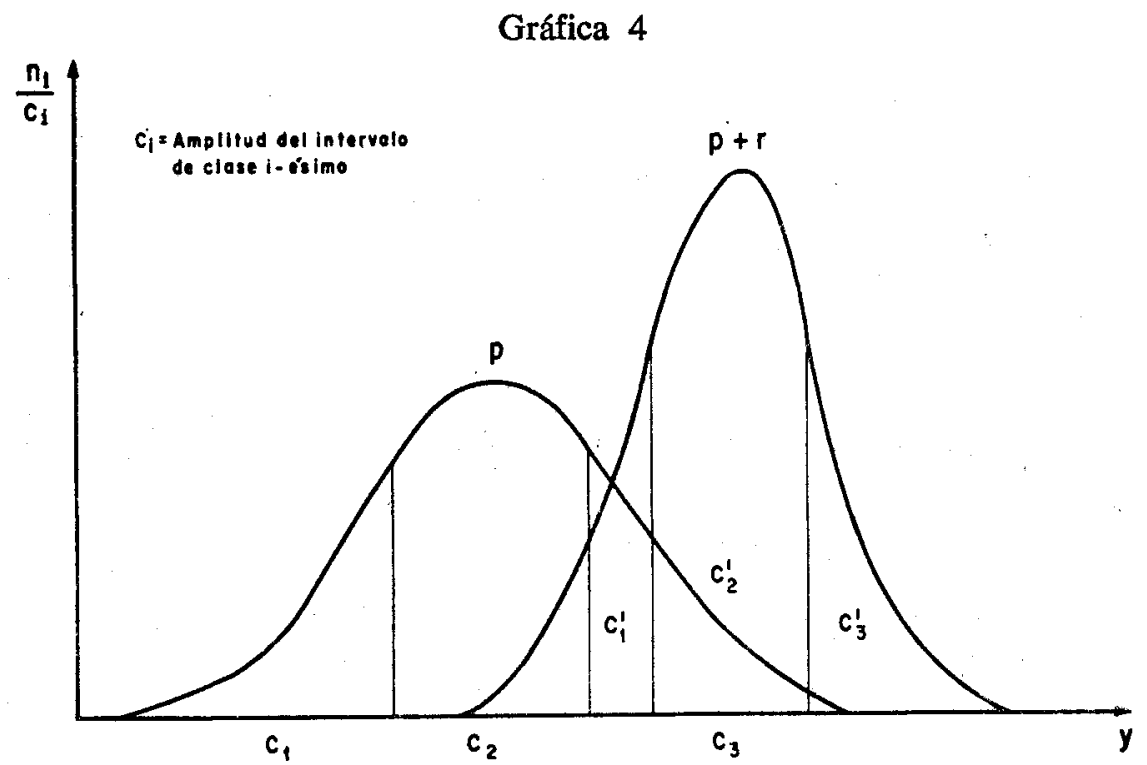


elevada que la de $p$, presenta un menor nivel de concentración (los valores de la variable están más cerca de la media). En este movimiento general se puede reconocer un acercamiento de las medias de las clases $C_{1}^{\prime}, C_{2}^{\prime}$ y $C_{3}^{\prime}$ a la media general (lo que implica una tendencia a la iguación en la distribución del ingreso interclases); y además una clara propensión a la equidistribución al interior de cada clase. Estos movimientos conjugados resultan en una concentración menor en $p+r$ que en $p$.

Como se puede advertir con facilidad al examinar la ecuación (9), la descomposición del cambio de la varianza es sensible a efectos de escala. Por ello parece recomendable, antes de proceder a la resta de las ecuaciones (7) y (8), dividir cada una de ellas por el cuadrado de la media aritmética general de cada distribución. De esta manera se definen las igualdades:

$$
\begin{aligned}
& \frac{S_{p}^{2}}{\bar{Y}_{p}^{2}}=\frac{S_{d, p}^{2}}{\bar{Y}_{p}^{2}}+\frac{S_{e, p}^{2}}{\bar{Y}_{p}^{2}} \\
& \frac{S_{p+r}^{2}}{\bar{Y}_{p+r}^{2}}=\frac{S_{d, p+r}^{2}}{\bar{Y}_{p+r}^{2}}+\frac{S_{e, p+r}^{2}}{\bar{Y}_{p+r}^{2}}
\end{aligned}
$$

Para simplificar la notación definamos:

$$
\begin{aligned}
& \frac{S_{p}^{2}}{\bar{Y}_{p}^{2}}=W_{p} ; \frac{S_{d, p}^{2}}{\bar{Y}_{a}^{2}}=W_{d, p} ; \frac{S_{e, p}^{2}}{\bar{Y}_{p}^{2}}=W_{e, p} \\
& \frac{S_{p+r}^{2}}{\bar{Y}_{p+r}^{2}}=W_{p+r} ; \frac{S_{d, p+r}^{2}}{\bar{Y}_{p+r}^{2}}=W_{d, p+r} ; \frac{S_{e, p+r}^{2}}{\bar{Y}_{p+r}^{2}}=W_{e, p+r}
\end{aligned}
$$

Al reemplazar estas expresiones en (10) y (11) y restar (11) a (10) se llega a:

$$
W_{p}-W_{p+r}=\left(W_{d, p}-W_{d, p+r}\right)+\left(W_{e, p}-W_{e, p+r}\right)
$$

La interpretación de esta ecuación es absolutamente equivalente a la de la igualdad (9), ya que sólo se ha introducido un factor de corrección para eliminar la sensibilidad a cambios de escala.

Además, se sabe por la ecuación (4) que la intravarianzas totales en los momentos $p$ y $p+r$, corregidas por el efecto escala, responderán a:

$$
W_{d, p}=\frac{S_{d, p}^{2}}{\bar{Y}_{p}^{2}}=\frac{\sum_{g=1}^{G} S_{d, g, p}^{2} h_{g, p}}{\bar{Y}_{p}^{2}}
$$




$$
W_{d, p+r}=\frac{S_{d, p+r}^{2}}{\bar{Y}_{p+r}^{2}}=\frac{\sum_{\substack{g \\ p}}^{G} S_{d, g, p+r}^{2} h_{g, p+r}}{\bar{Y}_{p+r}^{2}}
$$

La diferencia entre ambas ecuaciones se puede descomponer de la siguiente manera:

$$
\begin{array}{cc}
W_{d, p}-W_{d, p+r}=\left(W_{d, 1, p}-W_{d, 1, p+r}\right)+\left(W_{d, 2, p}-W_{d, 2, p+r}\right)+\ldots \\
\ldots+\left(W_{d, G, p}-W_{d, G, p+r}\right) & (g=1,2, \ldots G) \\
\text { donde } W_{d, g, p}=\frac{S_{d, g, p}^{2} h_{g, p}}{\bar{Y}_{p}^{2}} & (g=1,2, \ldots G) \\
\mathrm{Y} \quad W_{d, g, p+r}=\frac{S_{d, g, p+r}^{2} h_{g, p+r}}{\bar{Y}_{p+r}^{2}} &
\end{array}
$$

En la ecuación (15) se ha tenido cuidado de hacer la diferencia sobre los términos correspondientes, es decir, sólo sobre los componentes definidos para cada clase en los tiempos $p$ y $p+r$.

La igualdad (15) resulta ser una descomposición del primer término encerrado entre paréntesis en el lado derecho de la ecuación (12) y por lo tanto se puede interpretar como el aporte que realiza cada grupo a la variación de la intravarianza total.

Aun cuando la apariencia de estas ecuaciones resulta complicada, las ideas que expresan son en extremo simples. Un ejemplo numérico acerca del tipo de resultados que surgen de la aplicación de estas ecuaciones permitirá mostrar de manera sencilla el alcance de estas expresiones formales.

Supóngase que la información se ha dividido en tres grupos; que se han realizado los cálculos de todos los términos $W$ en los instantes $p$ y $p+r$ y que al reemplazar los valores correspondientes en la ecuación (12) se llega al siguiente resultado:

$$
0.4=0.3+0.1
$$

Esto quiere decir que ha habido una tendencia a la igualación en la distribución del ingreso en el lapso transcurrido entre $p$ y $p+r$ (el valor es positivo porque la variabilidad en $p$ es mayor que en $p+r$ ); es decir, la concentración en este segundo momento es menor que en el primero). De este cambio global un $75 \%(0.3 / 0.4)$ es debido a una desconcentración del ingreso dentro de los grupos y sólo un 25\% (0.1/0.4) a una menor dispersión de las medias de los grupos con respecto a la media general. 
La ecuación (15) a su vez permite la descomposición de la variabilidad intragrupal. Así, conforme al ejemplo, supóngase que la ecuación (15) origina los siguientes valores:

$$
0.3=0.05+0.10+0.15 \text {. }
$$

Esta igualdad indica que el primer grupo aporta $1 / 6(0.05 / 0.30)$ al cambio de la intravarianza total entre $p$ y $p+r$. Los grupos 2 y 3 aportan $1 / 3(0.10 / 0.30)$ y $1 / 2(0.15 / 0.30)$, respectivamente, a esta variación.

Sería conveniente disponer de una ecuación de la misma naturaleza para descomponer la intervarianza. Esta igualdad se puede obtener a partir de las ecuaciones (6) y (9), corrigiendo por el cuadrado de la media. De esta manera se concluye que:

$$
\begin{aligned}
& W_{e, p}-W_{e, p+r}=\left(W_{e, 1, p}-W_{e, 1, p+r}\right)+\left(W_{e, 2, p}-W_{e, 2, p+r}\right)+\ldots \\
& \ldots+\left(W_{e, G, p}-W_{e, G, p+r}\right)
\end{aligned}
$$

donde $W_{e, g, p}=\frac{S_{e, g, p}^{2}}{\overline{Y_{p}^{2}}}$

$$
\begin{aligned}
& (g=1,2, \ldots G) \\
& (g=1,2, \ldots G)
\end{aligned}
$$$$
\text { y } \quad W_{e, g, p+r}=\frac{S_{e, g, p+r}^{2}}{\bar{Y}_{p+r}^{2}}
$$

Los términos entre paréntesis de la ecuación (16) indican el aporte que hace cada uno de los $G$ grupos al cambio de la intervarianza.

Conforme al ejemplo, se sabía que el aporte de la variación de la intervarianza a la desconcentración del ingreso era igual a 0.1. Supóngase que al realizar las operaciones indicadas en (16) se llega a los siguientes resultados:

$$
0.1=0.025+0.035+0.040
$$

Esto significa que de la contribución total que hace el cambio de la intervarianza al proceso de democratización en la distribución del ingreso, un $25 \%$ corresponde al primer grupo, un $35 \%$ al segundo y un $40 \%$ al tercero.

Los resultados de la aplicación del conjunto de ecuaciones desarrolladas pueden presentarse de manera resumida en un cuadro que sintetiza la información manejada (véase el cuadro 4).

Los resultados de las dos primeras columnas numéricas del cuadro 4 ya han sido comentados y el tipo de análisis a que dan lugar es indepen- 


\section{Cuadro 4}

DESCOMPOSICIÓN DEL CAMBIO DE LA VARIABILIDAD TOTAL

\begin{tabular}{cccc}
\hline Grupos & Intravariabilidad & Intervariabilidad & Total \\
\hline$G_{1}$ & 0.050 & 0.025 & 0.075 \\
$G_{2}$ & 0.100 & 0.035 & 0.135 \\
$G_{3}$ & 0.150 & 0.040 & 0.190 \\
Total & 0.300 & 0.100 & 0.400 \\
\hline
\end{tabular}

diente del número de grupos considerados. En consecuencia, no se abunda en mayores detalles, especialmente porque en la próxima sección se presenta un análisis bastante más pormenorizado. La tercera columna puede ser interpretada como el aporte total que hace cada une de los $G$ grupos al cambio en la concentración de la distribución. Este resultado tiene una justificación algebraica a través de la suma término a término de los elementos de las ecuaciones (15) y (16):

$$
\begin{aligned}
& W_{p}-W_{p+r}=\left(W_{d, p}-W_{d, p+r}\right)+\left(W_{e, p}-W_{e, p+r}\right)= \\
& =\left[\left(W_{d, 1, p}-W_{d, 1, p+r}\right)+\left(W_{e, 1, p}-W_{e, 1, p+r}\right)\right]+ \\
& +\left[\left(W_{d, 2, p}-W_{d, 2, p}-W_{d, 2, p+r}\right)+\left(W_{e, 2, p}-W_{e, 2, p+r}\right)\right]+\ldots \\
& +\left[\left(W_{d, G, p}-W_{d, G, p+r}\right)+\left(W_{e, G, p}-W_{e, G, p+r}\right)\right]
\end{aligned}
$$

Los términos encerrados por los paréntesis cuadrados son los aportes que cada grupo hace al cambio de la variabilidad total. En el cuadro 4 se observa que el grupo 1 aporta un $18.75 \%$ al cambio de la concentración, mientras que el aporte de los grupos 2 y 3 es de $33.75 \%$ y $47.50 \%$, respectivamente.

El mismo cuadro señala también que se puede evaluar —dentro del aporte que cada grupo ha hecho al cambio en la concentración- la contribución relativa de la intra y de la intervariabilidad. Así por ejemplo, del aporte de 0.190 que hace el grupo 3 al cambio en la concentración total, un 78.95\% (0.150) se debe al proceso de igualación ocurrido en su interior entre $p$ y $p+r$.

Por último, sería conveniente agregar a la propiedad de descomposición del cambio de las varianzas, la aditividad en el tiempo. Para analizar esta propiedad agreguemos a los momentos $p$ y $p+r$ el instante $p+r+s$ y apliquemos la ecuación (12) a pares consecutivos en el tiempo. La comparación entre $p$ y $p+r$ es igual a:

$$
W_{p}-W_{p+r}=\left(W_{d, p}-W_{d, p+r}\right)+\left(W_{e, p}-W_{e, p+r}\right)
$$


Al comparar $p+r$ con $p+r+s$ se tiene:

$$
W_{p+r}-W_{p+r+s}=\left(W_{d, p+r}-W_{d, p+r+s}\right)+\left(W_{e, p+r}-W_{e, p+r+s}\right)
$$

Al sumar miembro a miembro ambas ecuaciones se llega a:

$$
W_{p}-W_{p+r+s}=\left(W_{d, p}-W_{d, p+r+s}\right)+\left(W_{e, p}-W_{e, p+r+s}\right)
$$

$\mathrm{Al}$ examinar la estructura de las ecuaciones se puede advertir que si se hace el cálculo entre los instantes $p y p+r+s$ y entre $p$ y $p+r$, podríamos obtener la variación en la concentı ación entre $p+r$ y $r+s$ restando al cambio ocurrido entre $p$ y $p+r+s$ el valor del cambio entre $p$ y $p+r$. En síntesis, se concluye que las agualdades que permiten analizar la variación en la concentración son aditivas a través del tiempo.

La extensión de la aditividad en el tiempo a un número cualquiera de instantes o lapsos es inmediata y no presenta ninguna complicación adicional a las que ya se han considerado.

La propiedad de aditividad es independiente del hecho que las distribuciones se ubiquen en el tiempo o en el espacio. Si se compara la distribución de una variable entre países o regiones no habría en principio ninguna dificultad para calcular la diferencia en la concentración entre dos unidades cualesquiera conociendo las diferencias entre los pares restantes. Esta propiedad aplicada sobre el espacio se puede generalizar a un número cualquiera de comparaciones entre unidades.

En la siguiente sección se presenta un ejemplo de aplicación de las ideas desarrolladas en este parágrafo con datos construidos a partir de información estadística real.

\section{UN EJEMPLO NUMÉRICO DE APLICACIÓN DE LA DESCOMPOSICIÓN DE LA VARIANZA AL CAMBIO DE LA CONCENTRACIÓN ${ }^{10}$}

La información que trabajaremos en esta sección se encuentra en la recopilación realizada por Arturo León ${ }^{11}$ de datos relativos a la distribución del ingreso personal en Santiago de Chile para el periodo 1970-1973. La utilización que se hace de esta información sólo cumple fines ilustrativos. No se pretende discutir acerca de las características de los modelos sociales, políticos y económicos en los cuales se inscribe el problema de la distribución del ingreso. ${ }^{12}$

10. Todos los resultados que se presentan en esta sección han sido computados con calculadoras de bolsillo. Por lo tanto el lector encontrará pequeñas diferencias en las cifras que derivan de las aproximaciones efectuadas.

11 A. León, "Antecedentes para el análisis de la distribución personal y familiar del ingreso. Chile, 1970-1973", PROELCE, 1976.

12 El trabajo de J. Serra y A. León, "La redistribución del ingreso en Chile durante el gobierno de la Unidad Popular. Exito y frustración". (FLACSo-Santiago, 
Los cuadros estadísticos sobre la distribución del ingreso personal que figuran en la recopilación de Arturo León clasifican la información en seis categorías ocupacionales $(G=6)$ : obreros, empleados, trabajadores por cuenta propia, empleadores, fuerzas armadas y empleados domésticos. En el interior de cada una de estas categorías presentan la distribución del ingreso personal por tramos definidos en función de sueldos vitales. La información estadística detallada sobre la cual se basa el análisis presente se incluye en el Anexo 1.0

En el cuadro 5 se presenta la descomposición de la varianza total en intra e intervarianzas para los tres años considerados. ${ }^{14}$

\section{Cuadro 5}

VARIANZA TOTAL: INTER E INTRAVARIANZA

\begin{tabular}{|c|c|c|c|c|}
\hline Varinnzas inter & e intragrupos & 1970 & 1971 & 1972 \\
\hline $\begin{array}{l}\text { Intravarianza } \\
\text { Intervarianza }\end{array}$ & & $\begin{array}{lll}3 & 994 & 510.0 \\
1 & 704 & 891.3\end{array}$ & $\begin{array}{lll}5 & 733 & 163.1 \\
1 & 853 & 375.2\end{array}$ & $\begin{array}{lll}8 & 747 & 791.6 \\
2 & 705 & 095.6\end{array}$ \\
\hline Varianza. & & 5699401.3 & 7586538.3 & 11452887.2 \\
\hline
\end{tabular}

\section{Cuadro 6}

\section{INTRAVARIANZAS}

\begin{tabular}{lrrr}
\hline Categorias ocupacionales & \multicolumn{1}{c}{1970} & 1971 & \multicolumn{1}{c}{1972} \\
\hline Obreros & 89742.9 & 128143.8 & 308175.4 \\
Empleados & 2335326.1 & 3904710.6 & 5559290.8 \\
Trabajadores por cuenta & 644076.0 & 981457.0 & 1886414.4 \\
propia & 884104.8 & 665615.4 & 883073.7 \\
Empleadores & 12507.1 & 40409.7 & 64165.7 \\
Fuerzas arnadas & 28753.1 & 12826.6 & 46671.4 \\
Empleados domésticos & 3994510.0 & 5773163.1 & 8747791.6 \\
Total & & & \\
\hline
\end{tabular}

1978) es un primer intento por vincular los cambios en la distribución del ingreso ocurridos en Chile durante 1970 y 1973 con las políticas económicas.

${ }^{13}$ En los cálculos no se ha considerado el número de desocupados, de manera principal por carecer de los datos adecuados. En sentido estricto en un estudio que pretenda analizar el proceso real de concentración del ingreso habría que incluir una categoría con las personas a quienes corresponde un ingreso nulo.

14 Los cuadros que incluimos en esta sección son resultados parciales de los procedimientos de cómputo que se detallan en el Anexo 2, de conformidad con los desarrollos algebraicos presentados en la sección anterior. 
Los totales que se presentan en la primera línea del cuadro 5 , se forman por la suma de los aportes que hacen cada una de las categorías ocupacionales. Así por ejemplo, la intravarianza total para el año 1971 es igual al total de la columna correspondiente del cuadro 6 .

La importancia relativa de cada categoría ocupacional dentro del total de cada año mide la contribución que realiza a la intravarianza de ese año. De la misma manera se forma la línea correspondiente a la intervarianza en el cuadro 5: por ejemplo, el valor numérico de la intravarianza en 1972 es igual a la suma de la tercera columna del cuadro 7 .

\section{Cuadro 7}

INTERVARIANZAS

\begin{tabular}{|c|c|c|c|c|c|c|}
\hline Categorias ocupacionales & & 970 & & 1971 & & 972 \\
\hline obreros & 249 & 318.5 & 411 & 723.6 & 692 & 684.5 \\
\hline Empleados & 165 & 913.5 & 448 & 156.5 & 547 & 530.9 \\
\hline $\begin{array}{l}\text { Trabajadores por cuenta } \\
\text { propia }\end{array}$ & 5 & 449.9 & 37 & 184.0 & 22 & 762.5 \\
\hline Empleadores & 1. 180 & 727.9 & 727 & 545.3 & 1080 & 073.0 \\
\hline Fuerzas armadas & & 415.6 & 4 & 226.5 & 20 & 842.7 \\
\hline Empleados domésticos & 103 & 065.9 & 224 & 539.3 & 341 & 202.0 \\
\hline Total & 1704 & 891.3 & 1853 & 375.2 & 2705 & 095.6 \\
\hline
\end{tabular}

La importancia relativa de cada casilla dentro del total de la columna indica el aporte de cada categoría ocupacional a la intervarianza del año.

El cuadro 8 se obtiene a través de la suma de los valores que figuran en las casillas correspondientes de los cuadros 6 y 7. Así por ejemplo el valor de la casilla definida por la categoría "obreros" y el año 1970, se

\section{Cuadro 8}

VARIANZA TOTAL: CATEGORÍAS OCUPACIONALES

\begin{tabular}{lrrr}
\hline Categorías ocupacionales & \multicolumn{1}{c}{1970} & \multicolumn{1}{c}{1971} & \multicolumn{1}{c}{1972} \\
\hline obreros & 339061.4 & 539867.4 & 1000859.9 \\
Empleados & 2501239.6 & 4362867.1 & 6106821.7 \\
Trabajadores por cuenta & 649525.9 & 1018641.0 & 1909177.1 \\
propia & 2064832.7 & 1393160.7 & 1963146.7 \\
Empleadores & 12922.7 & 44636.2 & 85008.4 \\
Fuerzas armadas & 131819.0 & 237365.9 & 387873.4 \\
Empleados domésticos & 5699401.3 & 7586538.3 & 11452887.2 \\
Total & & & \\
\hline
\end{tabular}


obtiene sumando los resultados que para la misma figuran en los cuadros de intra e intervarianzas.

Una característica notable de la información provista por el cuadro 8 es el elevado aporte que hacen a la varianza total, para todos los años, las categorías "empleados" y "empleadores". Este hecho puede ser una consecuencia tanto de un problema en la definición de estas clases como de un efecto de escala.

Al examinar la información contenida en los cuadros 6 y 7 se aprecia que el aporte que hace la categoría "empleados" a la varianza total (1970: 2501239.6 ; 1971: $4352867.1 ; 1972: 6106821.7$ ) se debe de manera fundamental a un problema en su definición. Esta clase estadística parece estar compuesta por un amplio espectro de grupos de ingreso, lo que se refleja en la importancia relativa de las intravarianzas: 2335326.1 / 2501239.6 (93.37\%), $3904710.6 / 4352867.1$ (89.7\%), $5559290.8 /$ $6106821.7(91.03 \%)$, para los años 1970, 1971 y 1972, respectivamente.

En el caso de la categoría "empleadores" el alto valor de la varianza se debe primordialmente a las intervarianzas. La importancia relativa de las intervarianzas alcanza los valores $57.2 \%$; $57.2 \%$ y $55.2 \%$, para los años 1970,1971 y 1972, respectivamente. Esto significa que el aporte de esta clase estadística proviene fundamentalmente de la ubicación de su media con respecto a la media general de la distribución.

Queda claro entonces que la contribución significativa que hacen ambas categorías a la varianza total difiere en su composición. En el caso de los empleados parece evidente que existe un problema de heterogeneidad, mientras que en el caso de los empleadores el aporte del sector pareciera ser genuino.

En cuanto a las otras categorías ocupacionales podría hacerse un análisis semejante. En términos generales se encuentra también un problema de definición en las categorías "trabajadores por cuenta propia" y "fuerzas armadas", no aconteciendo lo mismo con los restantes grupos.

Por otra parte, la comparación de las varianzas a través de los años indica un fuerte crecimiento de la varianza total para todas las categorías ocupacionales. Esta situación es un reflejo nítido de un efecto de escala. Es conocido el proceso hiperinflacionario que afectó a Chile durante el último decenio. Los datos incorporan el efecto de este proceso.

Respecto a los problemas de definición de las distintas categorías ocupacionales no es posible afinar la información. El efecto escala en cambio se corrige - de acuerdo con las ecuaciones de la sección precedentedividiendo los datos de cada año por el cuadrado de su correspondiente media general, los cuales resultan ser: 2916922.4 en 1970; 5998090.8 en 1971 y 12215724.0 en 1972.

Los cuadros $9,10,11$ y 12 contienen los valores corregidos por el efecto escala. Estos cuadros constituyen insumos necesarios para proceder al análisis de los cambios experimentados por la concentración del ingreso. Aun cuando en sí mismos pueden ser utilizados para estudiar una 
serie de características de la distribución del ingreso personal en Santiago de Chile en el periodo 1970-1972, en este trabajo no se aborda el examen de la información desde este punto de vista. Sólo preocupa mostrar las bondades analíticas del método sugerido para examinar los cambios ocurridos en la concentración.

\section{Cuadro 9}

VARIANZA TOTAL CORREGIDA POR EFECTO DE ESCALA

(Variabilidad total)

\begin{tabular}{lccc}
\hline Inter e intra varianzas & 1970 & 1971 & 1972 \\
\hline Intravarianza & 1.374 & 0.957 & 0.719 \\
Intervarianza & 0.586 & 0.309 & 0.222 \\
Varianza & 1.960 & 1.267 & 0.941 \\
\hline
\end{tabular}

Cuadro 10

INTRAVARIANZAS CORREGIDAS POR EFECTO DE ESCALA

(Intravariabilidad)

\begin{tabular}{lccc}
\hline \multicolumn{1}{c}{ Categorias ocupacionales } & 1970 & 1971 & 1972 \\
\hline Obreros & 0.031 & 0.021 & 0.024 \\
Empleados & 0.803 & 0.657 & 0.457 \\
Trabajadores por cuenta propia & 0.222 & 0.164 & 0.155 \\
Empleadores & 0.304 & 0.111 & 0.073 \\
Fuerzas armadas & 0.004 & 0.007 & 0.005 \\
Eapleados donésticos & 0.010 & 0.002 & 0.094 \\
Total & 1.374 & 0.957 & 0.719 \\
\hline
\end{tabular}

\section{Cuadro 11}

INTERVARIANZAS CORREGIDAS POR EFECTO DE ESCALA

(Intervariabilidad)

\begin{tabular}{lccc}
\hline Categorías ocupacionales & 1970 & 1971 & 1972 \\
\hline & & & \\
Obreros & 0.086 & 0.069 & 0.057 \\
Empleados & 0.057 & 0.075 & 0.045 \\
Trabajadores por cuenta propia & 0.002 & 0.006 & 0.002 \\
Empleadores & 0.406 & 0.121 & 0.089 \\
Fuerzas armadas & 0.000 & 0.001 & 0.002 \\
Enpleados domêsticos & 0.055 & 0.037 & 0.023 \\
Total & 0.586 & 0.310 & 0.223
\end{tabular}


Cuadro 12

VARIABILIDAD total: Categorías OCUPACionales

\begin{tabular}{|c|c|c|c|}
\hline Categorías ocupacionales & 1970 & 1971 & 1972 \\
\hline Obreros & 0.117 & 0.090 & 0.082 \\
\hline Limpleados & 0.860 & 0.727 & 0.502 \\
\hline Trubajadores nor cuenta propia & 0.223 & 0.170 & 0.157 \\
\hline Empleadores & 0.710 & 0.233 & 0.161 \\
\hline Fuevas armacis & 0.004 & 0.007 & 9.097 \\
\hline Eimplcados domésticos & 0.045 & 0.039 & 0.032 \\
\hline Total 1 & 1.959 & 1.267 & $0.9+1$ \\
\hline
\end{tabular}

La información provista por los cuadros 9 a 12 permite construir un cuadro resumen cuyos valores provienen de las restas de los elementos correspondientes de dichos cuadros (véase el cuadro 13).

\section{Cuadro 13}

DESCOMPOSICIÓN DEL CAMBIO DE LA VARIABILIDAD TOTAL DISTINGUIENDO ENTRE INTER E INTRAVARIABILIDAD CORREGIDAS POR EFECTO DE ESCALA

\begin{tabular}{|c|c|c|c|}
\hline & $1970-1971$ & $1971-1972$ & $1970-1972$ \\
\hline Intervariabilidad & 0.276 & 0.087 & $\underline{0.363}$ \\
\hline $\begin{array}{l}\text { Obreros } \\
\text { Empleados } \\
\text { Trabajadores por cuenta propia } \\
\text { Empleadores } \\
\text { Fidras armadas } \\
\text { rmpleados domésticos }\end{array}$ & $\begin{array}{r}0.017 \\
-0.018 \\
-0.004 \\
0.284 \\
-0.001 \\
-0.002\end{array}$ & $\begin{array}{r}0.012 \\
0.030 \\
0.004 \\
0.033 \\
-0.001 \\
0.009\end{array}$ & $\begin{array}{r}0.029 \\
0.012 \\
0.000 \\
0.317 \\
.0 .002 \\
0.007\end{array}$ \\
\hline Intravariabilidal & 0.417 & $\underline{0.233}$ & $\underline{0.655}$ \\
\hline $\begin{array}{l}\text { Obreros } \\
\text { Empleados } \\
\text { Trabajadores por cuenta propia } \\
\text { Empleadores } \\
\text { Fuerzas armadas } \\
\text { Empleados domésticos }\end{array}$ & $\begin{array}{r}0.010 \\
0.151 \\
0.058 \\
0.193 \\
-0.003 \\
0.008\end{array}$ & $\begin{array}{r}-0.004 \\
0.195 \\
0.009 \\
0.038 \\
0.002 \\
-0.002\end{array}$ & $\begin{array}{r}0.006 \\
0.346 \\
0.067 \\
0.231 \\
-0.001 \\
0.006\end{array}$ \\
\hline Variabilidad total & 0.693 & $\underline{0.325}$ & 1.018 \\
\hline $\begin{array}{l}\text { Obreros } \\
\text { Empleados } \\
\text { Trabajadores por cuenta propia } \\
\text { Empleadores } \\
\text { Fuerzas armidas } \\
\text { Empleados donesticos }\end{array}$ & $\begin{array}{r}0.027 \\
0.133 \\
0.053 \\
0.477 \\
-0.003 \\
0.006\end{array}$ & $\begin{array}{l}0.008 \\
0.225 \\
0.013 \\
0.072 \\
0.000 \\
0.007\end{array}$ & $\begin{array}{r}0.055 \\
0.358 \\
0.066 \\
0.549 \\
-0.003 \\
0.013\end{array}$ \\
\hline Variabilidad total. & 0.693 & $\underline{0.325}$ & 1.018 \\
\hline Intervariabilidad & 0.276 & 0.087 & 0.363 \\
\hline Intravariabilidad & 0.417 & 0.233 & 0.655 \\
\hline
\end{tabular}


El cuadro 13 está compuesto por cuatro secciones. Las tres primeras se refieren a la descomposición de la variabilidad total en intra e intervariabilidad, distinguiendo por categoría ocupacional. La última parte muestra el cambio de la variabilidad total separando el aporte de la inter y de la intravariabilidad.

Para ilustrar en sus líneas generales la potencialidad analítica del instrumento, se empieza el análisis por la última sección del cuadro.

Es claro que en el periodo ha habido una tendencia sistemática a la distribución del ingreso cada vez más igualitaria (los valores de la variabilidad total son positivos porque la de cada año consecutivo es menor, es decir, la dispersión en torno a la media disminuye). El impacto más fuerte se produjo en el año 1971 con respecto a 1970.

Por otro lado, la distribución más equitativa del ingreso en 1971 en relación a 1970 en un $39.8 \%(0.276 / 0.693)$ a la intervariabilidad y en un $60.2 \%(0.417 / 0.693)$ a la intravariabilidad; el cambio experimentado en 1972 con respecto a 1971 reconoce también como fuente principal a la intravariabilidad $(73.2 \%)$ y en forma secundaria a la intervariabilidad (26.8\%). En el periodo considerado (1970-1972) la reducción de la variabilidad total se explica en un $64.3 \%$ por lo ocurrido con la intravariabilidad y en un $35.7 \%$ por la alteración en la intervariabilidad.

Estas características asociadas a los cambios en las distribuciones de frecuencias señalan que en el caso analizado, la tendencia a la igualación en la distribución del ingreso entre 1970 y 1972 se descompone en dos tipos de movimientos: en primer lugar - por su importancia relativa mayor- una clara tendencia en favor de la equidistribución en el interior de las categorías ocupacionales; en segundo lugar -aunque de importancia también sensible - un movimiento de igualación entre las categorías ocupacionales (acercamiento de las medias de las clases estadísticas a la media total).

Sin embargo, esta información no indica si dichos movimientos son homogéneos para todas las clases estadísticas o si se producen sólo en algunas de ellas. A raíz de esta insuficiencia es que en el cuadro 13 se han incluido las tres secciones superiores, referidas precisamente a la descomposición de la variabilidad total en intra e intervariabilidad, pero reconociendo el aporte de cada una de las categorías ocupacionales.

La contribución más significativa al cambio en la variabilidad total entre 1970 y 1971 corresponde a la categoría ocupacional "empleadores", la cual aporta un $68.8 \%(0.477 / 0.693)$. Sin embargo, para el periodo 1971-1972 el aporte más importante es hecho por los empleados -con un $69.2 \%$, seguido por empleadores con un $22.2 \%$. Estas tres categorías explican el $91.4 \%$ del aporte de los grupos ocupacionales al cambio en dirección a una distribución más equitativa del ingreso.

Debemos observar que la modificación más pronunciada hacia una equidistribución se produce entre 1970 y 1971, en comparación con 19711972. En consecuencia, el aporte que corresponde a los empleadores en el movimiento total del periodo $1970-1972$ es el de mayor relevancia 
(alcanza un $53.9 \%$ ), y queda en segundo lugar la contribución del sector "empleados" (35.2\%).

Llama también la atención en este cuadro la escasa participación en el movimiento de "democratización" de los ingresos de las categorías "obreros", "trabajadores por cuenta propia" y "empleados domésticos". Todos estos grupos ocupacionales tienen en común el hecho de participar en un movimiento general hacia la igualdad, exceptuándose sólo el sector "fuerzas armadas", donde se puede apreciar un leve desplazamiento en dirección a una mayor concentración.

En resumen, se observa que la distribución del ingreso personal en Santiago de Chile tendió, en el periodo 1970-1972 a una mayor equidad. Ahora bien, el análisis de la información relativa a la variabilidad total señala que este movimiento se explica de manera fundamental por el aporte que han realizado las categorías ocupacionales "empleadores", y "empleados". Sin embargo, el análisis de esta información no permite aún precisar si estas contribuciones se producen de manera sustancial por cambios en la distribución dentro de cada clase estadística o por una tendencia a la disminución de las diferencias de ingresos entre ellas.

Examinemos a continuación la forma como se descompone el aporte de los grupos ocupacionales más significativos, separando la parte que proviene de alteraciones en el interior de cada categoría de aquella que resulta de la modificación de su posición relativa.

La categoría ocupacional "empleadores" exhibe un doble movimiento hacia la igualdad "democrática". A simple vista se destaca que en el bienio 1970/1971 el aporte más importante al cambio tanto de la intra como de la intervariabilidad se debe a este grupo. Para el periodo 1971-1972 su contribución a la equidistribución se debe prioritariamente a la intervariabilidad, vale decir, a un acercamiento de su valor medio a la media general. Para el conjunto del periodo, o sea, para el trienio 1970/1972 el aporte de los empleadores resulta ser de la mayor importancia, tanto por un proceso de reducción de la dispersión al interior del grupo como por una caída sustancial de su ingreso en relación con los restantes grupos. Más adelante se examina con mayor detalle este segundo tipo de contribución al cambio en la concentración.

La incidencia de los empleados, no es de importancia en el bienio 1970/ 1971. Sí lo es en el periodo 1971-1972, en que ambas categorías determinan casi del todo el movimiento de la intravariabilidad. Sin embargo, difiere su participación en el cambio de la intervariabilidad, donde la contribución del sector "trabajadores por cuenta propia" es insignificante no así la de los empleados, que ocupan el segundo lugar después de los empleadores. Si el movimiento se refiere al periodo 1970-1972, la importancia del papel que han jugado los empleados y los trabajadores por cuenta propia se limita a una distribución del ingreso más equitativa en el interior de cada grupo.

Hasta ahora no se ha considerado la información referente a los sig- 
nos de los valores relativos a la intra e intervariabilidad consignados en el cuadro 13.

Como el signo de la intravariabilidad por categoría ocupacional (por ejemplo para la categoría "fuerzas armadas" en la columna 1970-1971) resulta de la diferencia entre las intravarianzas de 1970 y de 1971, un valor negativo significa que la intravariabilidad de 1971 es mayor que la de 1970. Si la varianza para las "fuerzas armadas" es mayor en 1971 que en 1970, esto significa una mayor dispersión, es decir, una mayor concentración de sus ingresos. Un signo positivo remitirá, por el contrario, a una tendencia a la igualación en el interior del grupo considerado. Así por ejemplo, los obreros experimentaron entre 1970 y 1971 una leve tendencia a la "democratización" interna de sus ingresos, mientras que en 19711972 se produjo un pequeño movimiento hacia la concentración. El resultado conjugado de estos dos movimientos produjo en definitiva una ligera "democratización" al interior de la categoría.

En síntesis, para la intravariabilidad una diferencia con signo positivo expresa una tendencia a la igualación interna, mientras que una con signo negativo refleja un movimiento hacia la concentración.

$\mathrm{La}$ información que provee el signo de la diferencia entre intervariabilidades no admite ser interpretada de manera tan inmediata.

En efecto, un valor negativo expresa que la intervariabilidad de, por ejemplo, 1971, es mayor que la de 1970, la cual se interpreta como un alejamiento de los valores medios de las categorías en relación a sus respectivas medias generales. Sin embargo, un signo negativo puede originarse de dos maneras distintas:

Dadas, por ejemplo, dos categorías ocupacionales, una por encima y la otra por debajo de la media general de la distribución en 1970, un alejamiento mayor con respecto a la media de la distribución en 1971 generará un valor con signo negativo en el cambio de la intervariabilidad. Pero en el caso del grupo ubicado originalmente (en 1970) encima de la media, se tratará de una mejora relativa de su posición, en tanto que la categoría situada en 1970 bajo la media habrá experimentado un empeoramiento relativo. ${ }^{15}$

Si el signo es positivo, se tiene una situación análoga: aun cuando la intervariabilidad ha disminuido, es decir, las medias de los grupos se han acercado a la media general, esto puede ser el resultado de un mejoramiento relativo de su posición (si el grupo originalmente estaba por debajo de la media general) o de un deterioro relativo (si estaba por encima de la media global).

La aplicación de estas ideas a los resultados del cambio en la intervariabilidad en el cuadro 13 permite construir los cuadros 14 y 15 que resumen la situación relativa de las seis categorías ocupacionales.

\footnotetext{
15 Obsérvese que el mejoramiento o empeoramiento en la posición de las categorías ocupacionales es relativo, por cuanto no es contradictorio un empeoramiento relativo con una mejora absoluta (alza de la media general).
} 


\section{Cuadro 14}

\section{Cambios en las posiciones relativas de las CATEGORÍAS OCUPACIONALES (1970-1971)}

\begin{tabular}{lll}
\hline Situación original & Mejoran & Empeoran \\
\hline Por abajo de la media general & Obreros $(+)$ & $\begin{array}{l}\text { Trabajadores por cuenta propia }(-) \\
\text { Empleados domésticos }(-)\end{array}$ \\
\hline Por encima de la media general & $\begin{array}{l}\text { Empleados }(-) \\
\text { Fuerzas amadas }(-)\end{array}$ & Empleadores $(+)$ \\
\hline
\end{tabular}

\section{Cuadro 15}

\section{Cambios en las posiciones Relativas de las CATEGORÍAS OCUPACIONALES (1971-1972)}

\begin{tabular}{|c|c|c|}
\hline Situación original & Mejoran & Empeoran \\
\hline Por abajo de la media general & $\begin{array}{l}\text { Obreros }(+) \\
\text { Trabajadores por cuenta propia }(+) \\
\text { Impleados domés ticos }(+)\end{array}$ & \\
\hline Por encima de la media general & Fuerzas armadas $(-)$ & $\begin{array}{l}\text { Empleados }(+) \\
\text { Empleadores }(+)\end{array}$ \\
\hline
\end{tabular}

El movimiento de los cambios en las intervariabilidades para el trienio 1970-1972 puede ser representado en el resumen que aparece en el cuadro 16.

\section{Cuadro 16}

\section{CAMBIOS EN LAS POSICIONES RELATIVAS DE LAS} CATEGORÍAS OCUPACIONALES (1970-1972)

\begin{tabular}{|c|c|c|}
\hline Situación original & Me joran & Einpeoran \\
\hline Por abajo de la media general & $\begin{array}{l}\text { Obreros (+) } \\
\text { Trabajadores por cuenta propia }(+) \\
\text { Empleados domés ticos }(+)\end{array}$ & \\
\hline Por cncimal de la media general & Fuerzas armadas $(-)$ & $\begin{array}{l}\text { Fimpleados }(+) \\
\text { Empleadores }\end{array}$ \\
\hline
\end{tabular}

Se considera que la estructura de los cuadros puede ser puesta en correspondencia con distintos momentos de la política social y económica que se vivieron en Chile entre los años 1970 y 1972. Es probable que no 
sólo estos cuadros, sino el conjunto de resultados presentados sirvan para interrogarse respecto a los cambios acontecidos durante este periodo. ${ }^{18}$

\section{Conclusiones}

Los desarrollos que se han presentado en las secciones precedentes se pueden ordenar alrededor del siguiente conjunto de ideas centrales:

1) El coeficiente de Gini, en su versión habitual, permite medir el grado o nivel de concentración de una distribución, pero no es sensible a cambios en su forma.

2) En la segunda sección se han ofrecido algunas indicaciones de naturaleza general, que a partir de la idea que individualiza al coeficiente de Gini, permite tomar en cuenta el hecho que dos distribuciones pueden presentar el mismo nivel de concentración aunque sus formas sean diferentes. En esta misma dirección se ha esbozado una posible solución mediante la construcción de un coeficiente Gini-intervalo.

3) Sin embargo, la estrategia propuesta no parece fácil de generalizar a distribuciones de frecuencias en las cuales la información ha sido clasificada de acuerdo a más de un criterio (aun cuando no se descarta la posibilidad de extender la definición del coeficiente de Gini a dos o más dimensiones). Así por ejemplo, si se tratase de estudiar la concentración de una distribución bidimensional de frecuencias se podría definir un coeficiente de Gini extendido, como la relación entre el volumen efectivo y el volumen máximo de concentración.

4) Cuando el interés del estudio radica en comprar dos o más distribuciones de frecuencias se podría aplicar la técnica de los coeficientes Giniintervalo, siempre que las distribuciones fuesen unidimensionales. En virtud de las consideraciones expuestas en el punto anterior, cuando se intenta comparar distribuciones de dos o más dimensiones, dicha técnica se vuelve en extremo compleja. Para este tipo de problemas es que proponemos recurrir al uso del teorema de descomposición de la varianza.

5) Si bien la técnica propuesta en la sección III no permite construir un indicador con límites conocidos del grado de concentración de la distribución, la simple propiedad de descomposición provee de un poderoso instrumento para estudiar los aportes de los distintos componentes del cambio total experimentado por la o las distribuciones.

6) En la cuarta sección se ha desarrollado un análisis a título de ejemplo, en que se hace un uso extensivo de la propiedad de descomposición. $\mathrm{La}$ ilustración se realizó con una distribución de frecuencias de ingreso personal, clasificada por categorías ocupacionales y por tramos de sueldos vitales. En términos metodológicos, el rasgo más relevante de este ejercicio consistió en la posibilidad de identificar la contribución que las dis-

16 El lector interesado encontrará en el artículo ya citado de J. Serra y A. León, un análisis más detenido de las políticas seguidas durante este periodo. 
tintas categorías ocupacionales han realizado al proceso tendiente a la equidistribución de la renta. Además se pudo señalar si dicho aporte se debía a un movimiento en el interior de las categorías ocupacionales (intravariabilidad) o a un desplazamiento de ellas con respecto a la media general (intervariabilidad).

7) En síntesis, en este trabajo se espera haber mostrado con claridad que el coeficiente de Gini es un buen instrumento para medir grado de concentración en distribuciones unidimensionales de frecuencias. Para el estudio comparativo de dos o más distribuciones pluridimensionales situadas en el tiempo o en el espacio, se propone recurrir al teorema de descomposición de la varianza. Su potencialidad reside en la capacidad de identificación de las distintas fuentes que componen la variación en los niveles generales de concentración. 
ANEXO 1

DISTRIBUCIÓN DEL INGRESO PERSONAL EN SANTIAGO DE CHILE, 1970-1972, POR CATEGORIAS OCUPACIONALES Y TRAMOS DE INGRESOS (en sueldos vitales). ${ }^{17}$

\section{Cuadro 1}

Gran Santiago: Distribución Del ingreso personal POR CATEGORÍAS OCUPACIONALES, 1970

\begin{tabular}{|c|c|c|c|c|c|c|}
\hline \multirow{2}{*}{ Categoría de ocupaciôn } & \multicolumn{2}{|c|}{ Perceptores } & \multirow{2}{*}{$\begin{array}{c}\text { Ingreso } \\
\text { tota1 } \\
\left(\begin{array}{l}0 \\
0\end{array}\right)\end{array}$} & \multirow{2}{*}{$\begin{array}{l}\text { Ingreso total } \\
\text { en cada } \\
\text { categorial }\end{array}$} & \multirow{2}{*}{$\begin{array}{l}\text { Ingreso } \\
\text { medio } \\
\text { al }\end{array}$} & \multirow{2}{*}{$\begin{array}{l}\text { Inoreso } \\
\text { nedio } \\
\text { relativo }\end{array}$} \\
\hline & Núnero & $\because$ & & & & \\
\hline Obreros & 1533 & 31.1 & 14.7 & 1240641 & 809.3 & 47.5 \\
\hline Enpleados & 1790 & 36.2 & 50.7 & 4262598 & 2381.3 & 139.7 \\
\hline $\begin{array}{l}\text { Trabajadores por cuenta } \\
\text { propia } \underline{\text { f }}\end{array}$ & 951 & 19.3 & 17.4 & 1461616 & 1536.9 & 90.1 \\
\hline Enpleadores & 115 & 2.3 & 12.1 & 1014593 & 8822.6 & 517.4 \\
\hline Fuerzas armadas & 43 & 0.9 & 0.9 & 82708 & 1923.4 & 112.8 \\
\hline Empleados domésticos & 502 & 10.2 & 4.2 & 350702 & 698.6 & $41 . n$ \\
\hline Total & 4934 & 100.0 & 100.0 & 8412358 & 1705.1 & \\
\hline
\end{tabular}

En escudos.

b Media $=100$.

c Trabajador independiente con no más de dos personas contratadas, excluidos los familiares no remunerados.

17 Información obtenida de A. León, "Antecedentes para el análisis de la distribución personal y familiar del ingreso. Chile 1970-1973". ProELCE, 197. 


\section{Cuadro 2}

Gran Santiago: Distribución del ingreso personal por tramos, EN SUELDOS VITALES, EN CADA CATEGORÍA OCUPACIONAL, 1970

\begin{tabular}{|c|c|c|c|c|c|c|c|}
\hline \multirow{2}{*}{$\begin{array}{c}\text { Tramo de } \\
\text { ingresos } \\
\text { a/ }\end{array}$} & \multicolumn{2}{|c|}{ Perceptores } & \multirow{2}{*}{$\begin{array}{l}\text { Ingreso } \\
\text { total } \\
\text { (s) }\end{array}$} & \multirow{2}{*}{$\begin{array}{r}\text { Ingreso } \\
\text { en cada } \\
\text { b/ }\end{array}$} & \multirow{2}{*}{$\begin{array}{l}\text { total } \\
\text { tramo }\end{array}$} & \multirow{2}{*}{\multicolumn{2}{|c|}{$\begin{array}{c}\text { Ingreso } \\
\text { medio } \\
\text { b/ }\end{array}$}} \\
\hline & Numero & : & & & & & \\
\hline \multicolumn{8}{|c|}{ OBREROS } \\
\hline $0.0 \mathrm{a} 0.5$ & 157 & 10.2 & 2.8 & 35 & .252 & & 224.5 \\
\hline 0.5 a 1.0 & 539 & 35.2 & 20.8 & $258^{\circ}$ & 721 & & 480.0 \\
\hline 1.0 a 1.5 & 404 & 26.3 & 25.2 & 312 & 597 & & 773.7 \\
\hline 1.5 a 2.5 & 311 & 20,3 & 30.0 & 372 & 065 & 1 & 196.3 \\
\hline 2.5 a 5.0 & 110 & 7,2 & 17.3 & 214 & 244 & 1 & 947.7 \\
\hline 5.0 a 10.0 & 11 & 0.7 & $\quad 3.2$ & 39 & 462 & 3 & 587.4 \\
\hline 10.0 a 20.0 & 1 & 0.1 & 0.7 & 8 & 300 & 8 & 300.0 \\
\hline más de 20.0 & 0 & 0.0 & 0.0 & & 0 & & 0.0 \\
\hline$T \circ t a t$ & 1533 & 100.0 & 100.0 & 1240 & 641 & & 809.3 \\
\hline \multicolumn{8}{|c|}{ EMPLEADOS } \\
\hline 0.0 a $0: 5$ & 36 & 2.0 & 0.2 & 7 & 409 & & 205.8 \\
\hline $0.5 \times 1.0$ & 189 & 10.6 & 2.3 & 99 & 766 & & 527.9 \\
\hline $1.0 \mathrm{a} 1.5$ & 249 & 13.9 & 4.5 & 194 & 123 & & 779.6 \\
\hline 1.5 a 2.5 & 447 & 25.0 & 12.9 & 548 & 178 & 1 & 226.3 \\
\hline 2.5 a 5.0 & 511 & 28.5 & 27.4 & 1162 & 400 & 2 & 274.9 \\
\hline 5.0 a 10.0 & 235 & 13.1 & 24.2 & 1033 & 479 & 4 & 397.8 \\
\hline 10.0 a 20.0 & 104 & 5.8 & 10.4 & 870 & 743 & 8 & 372.5 \\
\hline mâs de 20.0 & 19 & 1.1 & 8.1 & 346 & 400 & 18 & 231.6 \\
\hline$T \circ t a 1$ & 1790 & 100.0 & 100.0 & 4262 & 598 & 2 & 381.3 \\
\hline \multicolumn{8}{|c|}{ TRABAJADORES POR CUENTA PROPIA } \\
\hline 0.0 a 0.5 & 155 & 16.3 & 2.2 & 32 & 137 & & 207.3 \\
\hline 0.5 a 1.0 & 218 & 22.9 & 7.3 & 106 & 586 & & 488.9 \\
\hline $1.0 \mathrm{a} 1.5$ & 115 & 12.1 & 6.1 & 89 & 638 & 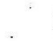 & 779.5 \\
\hline $1.5 \mathrm{a} 2.5$ & 169 & 17.8 & 14.1 & 206 & 626 & 1 & 222.6 \\
\hline $2.5 \mathrm{a} \cdot 5.0$ & 181 & 19.0 & 27.6 & 401 & 849 & 2 & 220.1 \\
\hline 5.0 a $10: 0$ & 78 & 8.2 & 22.0 & 321 & 650 & 4 & 123.7 \\
\hline 10.0 a 20.0 & 31 & 3.3 & 16.5 & 241 & .930 & 7 & 804.2 \\
\hline miás de 20.0 & 4 & 0.4 & 4.2 & .61 & 200 & 15 & 300.0 \\
\hline$T \circ t a I$ & 951. & 100.0 & .100 .0 & 1461 & 616 & 1 & 536.9 \\
\hline
\end{tabular}

an sueldos vitales.

b En escudos. 
Cuadro 3

Gran SANTiago: Distribución Del ingreso PERSONAL POR TRAMOS, EN SUELDOS VITALES, EN CADA CATEGORía OCUPACIONAL, 1970

\begin{tabular}{|c|c|c|c|c|c|c|c|}
\hline \multirow{2}{*}{$\begin{array}{l}\text { Trano de } \\
\text { ingresos } \\
\text { a/ }\end{array}$} & \multicolumn{2}{|c|}{ Perceptares } & \multirow{2}{*}{$\begin{array}{c}\text { Ingreso } \\
\text { total } \\
\text { (s) }\end{array}$} & \multirow{2}{*}{$\begin{array}{r}\text { Ingreso } \\
\text { en cada } \\
\text { b/ }\end{array}$} & \multirow{2}{*}{$\begin{array}{l}\text { total } \\
\text { tramo }\end{array}$} & \multirow{2}{*}{\multicolumn{2}{|c|}{$\begin{array}{l}\text { Ingreso } \\
\text { medio } \\
\text { b/ }\end{array}$}} \\
\hline & Número & $:$ & & & & & \\
\hline \multicolumn{8}{|c|}{ BMPLEADORES } \\
\hline 0.0 a 0.5 & 0 & 0.0 & 0.0 & & 0 & & 0.0 \\
\hline 0.5 a 1.0 & 2 & 1.7 & 0.1 & 1 & 000 & & 500.0 \\
\hline 1.0 a 1.5 & 0 & 0.0 & 0.0 & & 0 & & 0.0 \\
\hline 1.5 a 2.5 & 6 & 5.2 & 0.8 & 8 & 250 & 1 & 375.0 \\
\hline 2.5 a 5.0 & 19 & 16.5 & 4.8 & 48 & 300 & 2 & 542.1 \\
\hline 5.0 a 10.0 & 25 & 21.7 & 11.2 & 113 & 600 & 4 & 544.0 \\
\hline 10.0 a 20.0 & 33 & 28.8 & 29.1 & 295 & 043 & 8 & 940.7 \\
\hline mâs de 20.0 & 30 & 26.1 & 54.0 & 548 & 400 & 18 & 280.0 \\
\hline$T \circ t$ a 1 & 115 & 100.1 & 100.0 & 1014 & 593 & 8 & 822.5 \\
\hline \multicolumn{8}{|c|}{ FUERZAS ARMADAS } \\
\hline 0.0 a 0.5 & 0 & 0.0 & 0.0 & & 0 & & 0.0 \\
\hline 0.5 a 1.0 & 1 & 2.3 & 0.4 & & 350 & & 350.0 \\
\hline 1.0 a 1.5 & 8 & 18.6 & 7.3 & 6 & 080 & & 760.0 \\
\hline 1.5 a. 2.5 & 12) & 27.9 & 18.2 & 15 & 090 & 1 & 257.5 \\
\hline 2.5 a 5.0 & 17 & 39.6 & 44.9 & 37 & 088 & 2 & 181.6 \\
\hline 5.0 a 10.0 & 5 & 11.6 & 29.2 & 24 & 100 & 4 & 820.0 \\
\hline 10.0 a 20.0 & 0 & 0.0 & 0.0 & & 0 & & 0.0 \\
\hline más de 20.0 & 0 & 0.0 & 0.0 & & 0 & & 0.0 \\
\hline T. 0 t a 1 & 43 & 100.0 & 100.0 & 82 & 708 & 1 & 923.4 \\
\hline \multicolumn{8}{|c|}{ EMPLEADOS DOMESTICOS } \\
\hline $0.0 \cdot a 0.5$ & 56 & 11.2 & 3.9 & 13 & 596 & & 242.8 \\
\hline 0.5 a 1.0 & 180 & 35.9 & 24.6 & 86 & 211 & & 4.78 .9 \\
\hline 1.0 a 1.5 & 185 & 36.8 & 40.8 & 143 & 310 & & 774.6 \\
\hline 1.5 a 2.5 & 76 & 15.1 & 24.6 & 86 & 285 & & 135.3 \\
\hline 2.5 a 5.0 & 3 & 0.6 & 1.8 & 6 & 250 & 2 & 0.83 .3 \\
\hline 5.0 a 10.0 & 1 & 0.2 & 1.6 & 5 & 650 & 5 & 650.0 \\
\hline 10.0 a 20.0 & 1 & 0.2 & 2.7 & 9 & 400 & 9 & 400.0 \\
\hline más de 20.0 & $\mathbf{0}$ & 0.0 & 0.0 & & 0 & &, 0.0 \\
\hline Tota 1 & 502 & $10.0 . \dot{0}$ & 100.0 & 350 & 702 & & $69 \dot{8} .6$ \\
\hline
\end{tabular}

a En sueldos vitales.

b En escudo. 


\section{Cuadro 4}

Gran SANTIAgo: Distribución Del INGRESO PERsonal POR CATEGORÍAS OCUPACIONALES, 1971

\begin{tabular}{|c|c|c|c|c|c|c|}
\hline \multirow{2}{*}{ Categoría de ocupación } & \multicolumn{2}{|c|}{ Perceptores } & \multirow{2}{*}{$\begin{array}{l}\text { Ingreso } \\
\text { total } \\
(\&)\end{array}$} & \multirow{2}{*}{$\begin{array}{l}\text { Ingreso total } \\
\text { en cada a } \\
\text { categoria- }\end{array}$} & \multirow{2}{*}{$\begin{array}{l}\text { Ingreso } \\
\text { medio } \\
\text { (a) }\end{array}$} & \multirow{2}{*}{$\begin{array}{l}\text { Ingreso } \\
\text { nedio } \\
\text { relativo } \text { / }\end{array}$} \\
\hline & Nứnero & $:$ & & & & \\
\hline Obreros & 1362 & 26.3 & 13.0 & 1642220 & $1205: 7$ & 49.2 \\
\hline Empleados & 2081 & 40.2 & 57.5 & 7288840 & 3502.6 & 143.0 \\
\hline $\begin{array}{l}\text { Trabajadores por cuenta } \\
\text { propia c/ }\end{array}$ & 1037 & 20.0 & 16.5 & 2090561 & 20.16 .0 & 32.3 \\
\hline Empleadores & 127 & 2.5 & 7.9 & 1002300 & 7892.1 & 322.3 \\
\hline Fuerzas armadas & 55 & 1.1 & 1.3 & 169260 & 5077.4 & 125.7 \\
\hline Empleados domés ticos & 514 & 9.9 & 3.3 & $4847+2$ & 9.43 .1 & 33.5 \\
\hline Total & 5176 & 100.0 & 100.0 & 12677923 & 2449.0 & 100.0 \\
\hline
\end{tabular}

a En escudos.

b Media.

c Trabajador independiente con no más de dos personas contratadas, excluidos los familiares no remunerados. 


\section{Cuadro 5}

Gran Santiago: Distribución del ingreso personal por tramos, EN SUELDOS VITALES, EN CADA CATEGORÍA OCUPACIONAL, 1971

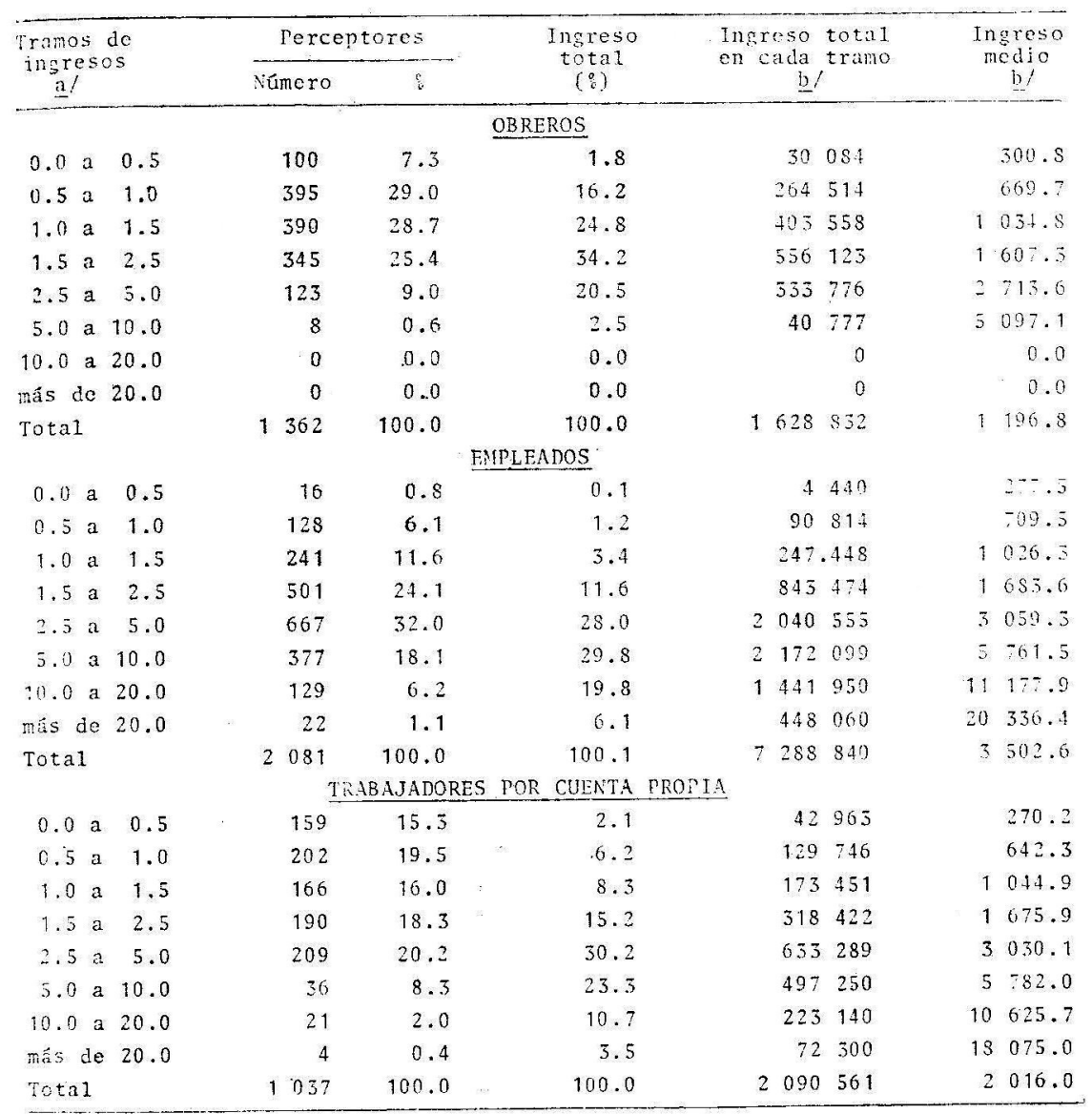

a En sueldos vitales.

b En escudos. 


\section{Cuadro 6}

Gran Santiago: Distribución del ingreso Personal por tramos, EN SUELDOS VITALES, EN CADA CATEGORÍA OCUPACIONAL, 1971

\begin{tabular}{|c|c|c|c|c|c|c|c|}
\hline \multirow{2}{*}{\multicolumn{2}{|c|}{$\begin{array}{c}\text { Tramos de } \\
\text { ingresos } \\
\text { a/ }\end{array}$}} & \multicolumn{2}{|c|}{ Perceptores } & \multirow{2}{*}{$\begin{array}{c}\text { Ingreso } \\
\text { total } \\
(\xi)\end{array}$} & \multirow{2}{*}{$\begin{array}{r}\text { Ingreso } \\
\text { en cada } \\
\text { b/ }\end{array}$} & \multirow{2}{*}{$\begin{array}{l}\text { total } \\
\text { tramo }\end{array}$} & \multirow{2}{*}{$\begin{array}{c}\text { Ingreso } \\
\text { medio } \\
\text { b/ }\end{array}$} \\
\hline & & Núne ro & 8 & & & & \\
\hline \multicolumn{8}{|c|}{ EMPLEADORES } \\
\hline $0.0 \mathrm{a}$ & 0.5 & 1 & 0.8 & $\cdot$ & & 400 & 400.0 \\
\hline $0.5 \mathrm{a}$ & 1.0 & 1 & 0.8 & - & & 300 & 800.0 \\
\hline $1.0 \mathrm{a}$ & 1.5 & 5 & 3.9 & 0.5 & 5 & 500 & 1100.0 \\
\hline $1.5 \mathrm{a}$ & 2.5 & 6 & 4.7 & 1.1 & 10 & 850 & 1808.8 \\
\hline $2.5 \mathrm{a}$ & 5.0 & 23 & 18.1 & 7.3 & 73 & 100 & 3178.3 \\
\hline $5.0 \mathrm{a}$ & 10.0 & 45 & 35.4 & 28.2 & 281 & 200 & 6248.9 \\
\hline $10.0 \mathrm{a}$ & 20.0 & 34 & 26.7 & 38.6 & 387 & 250 & 11389.7 \\
\hline más de & 20.0 & 12 & 9.4 & 24.3 & 243 & 200 & 20266.7 \\
\hline Total & & 127 & 100.0 & 100.0 & 1002 & 300 & 7892.1 \\
\hline \multicolumn{8}{|c|}{ FUERZAS ARMADAS } \\
\hline $0.0 \mathrm{a}$ & 0.5 & 0 & 0.0 & 0.0 & & $\mathbf{0}$ & 0.0 \\
\hline $0.5 \mathrm{a}$ & 1.0 & 0 & 0.0 & 0.0 & & 0 & 0.0 \\
\hline $1.0 \mathrm{a}$ & 1.5 & 11 & 20.0 & 6.5 & 10 & 985 & 996.6 \\
\hline $1.5 \mathrm{a}$ & 2.5 & 15 & 27.3 & 15.1 & 25 & 530 & 1702.0 \\
\hline $2.5 \mathrm{a}$ & 5.0 & 17 & 30.9 & 33.0 & 55 & 945 & 3290.9 \\
\hline $5.0 \mathrm{a}$ & 10.0 & 12 & 21.8 & 45.4 & 76 & 800 & 6400.0 \\
\hline $10.0 \mathrm{a}$ & 20.0 & 0 & 0.0 & 0.0 & & 0 & 0.0 \\
\hline más de & 20.0 & 0 & 0.0 & 0.0 & & 0 & 0.0 \\
\hline Total & & 55 & 100.0 & 100.0 & 169 & 260 & 3077,4 \\
\hline \multicolumn{8}{|c|}{ EMPLEADOS DOMESTICOS } \\
\hline $0.0 \mathrm{a}$ & 0.5 & 47 & $\cdot 9.1$ & 3.2 & 15 & 405 & 327.8 \\
\hline $0.5 \mathrm{a}$ & 1.0 & 166 & 32.3 & 22.7 & 110 & 273 & 664.3 \\
\hline $1.0 \mathrm{a}$ & 1.5 & 188 & 36.6 & 39.6 & 191 & 710 & 1019.7 . \\
\hline $1.5 \mathrm{a}$ & 2.5 & 112 & 21.8 & 33.9 & 164 & 504 & 1468.8 \\
\hline $2.5 \mathrm{a}$ & 5.0 & 1 & 0.2 & 0.6 & 2 & 850 & 2850.0 \\
\hline $5.0 \mathrm{a}$ & 10.0 & 0 & 0.0 & 0.0 & & 0 & 0.0 \\
\hline $10.0 \mathrm{a}$ & 20.0 & 0 & 0.0 & 0.0 & & 0 & 0.0 \\
\hline más de & 20.0 & 0 & 0.0 & 0.0 & & 0 & 0.0 \\
\hline Total & & 514 & 100.0 & 100.0 & 484 & 742 & 943.1 \\
\hline
\end{tabular}

an sueldos vitales.

b En escudos. 


\section{Cuadro 7}

Gran Santiago: Distribución del ingreso Personal por CATEGORÍAS OCUPACIONALES, 1972

\begin{tabular}{|c|c|c|c|c|c|c|}
\hline \multirow{2}{*}{ Categorias de ocupación } & \multicolumn{2}{|c|}{ Perceptores } & \multirow{2}{*}{$\begin{array}{c}\text { Ingreso } \\
\text { total } \\
\left(\begin{array}{l}0 \\
b\end{array}\right)\end{array}$} & \multirow{2}{*}{$\begin{array}{l}\text { Ingreso total } \\
\text { en cada a/ } \\
\text { categoria- }\end{array}$} & \multirow{2}{*}{$\begin{array}{l}\text { Ingreso } \\
\text { medio } \\
\text { af }\end{array}$} & \multirow{2}{*}{$\begin{array}{l}\text { Ingreso } \\
\text { medio } \\
\text { relativo }\end{array}$} \\
\hline & Núne ro & $\because$ & & & & \\
\hline Obreros & 1326 & 26.1 & 13.9 & $2466 \quad 656$ & 1860.2 & $5 \overline{3} .3$ \\
\hline Finpleados & 2135 & 42.1 & 55.9 & 9882352 & 4628.7 & 132.7 \\
\hline $\begin{array}{l}\text { Trabajadores por cuenta } \\
\text { propia cl }\end{array}$ & 1018 & 20.1 & 18.2 & 3208052 & 3151.3 & 90.5 \\
\hline Empleadores & 132 & 2.6 & 7.4 & 1310877 & 9930.9 & 284.7 \\
\hline Fuerzas amadas & 43 & 0.8 & 1.2 & 217418 & 5056.2 & 145.0 \\
\hline Empleados domesticos & 419 & 8.3 & 3.4 & 609906 & 1455.6 & 41.7 \\
\hline TotaI & 5073 & 100.0 & 100.0 & 17695261 & 3488.1 & 100.0 \\
\hline
\end{tabular}

a En escudos.

b Media.

c Trabajador independiente con no más de dos personas contratadas, excluidos los familiares no remunerados. 


\section{Cuadro 8}

Gran Santiago: Distribución del ingreso personal por tramos, EN SUELDOS VITALES, EN CADA CATEGORÍA OCUPACIONAL, 1972

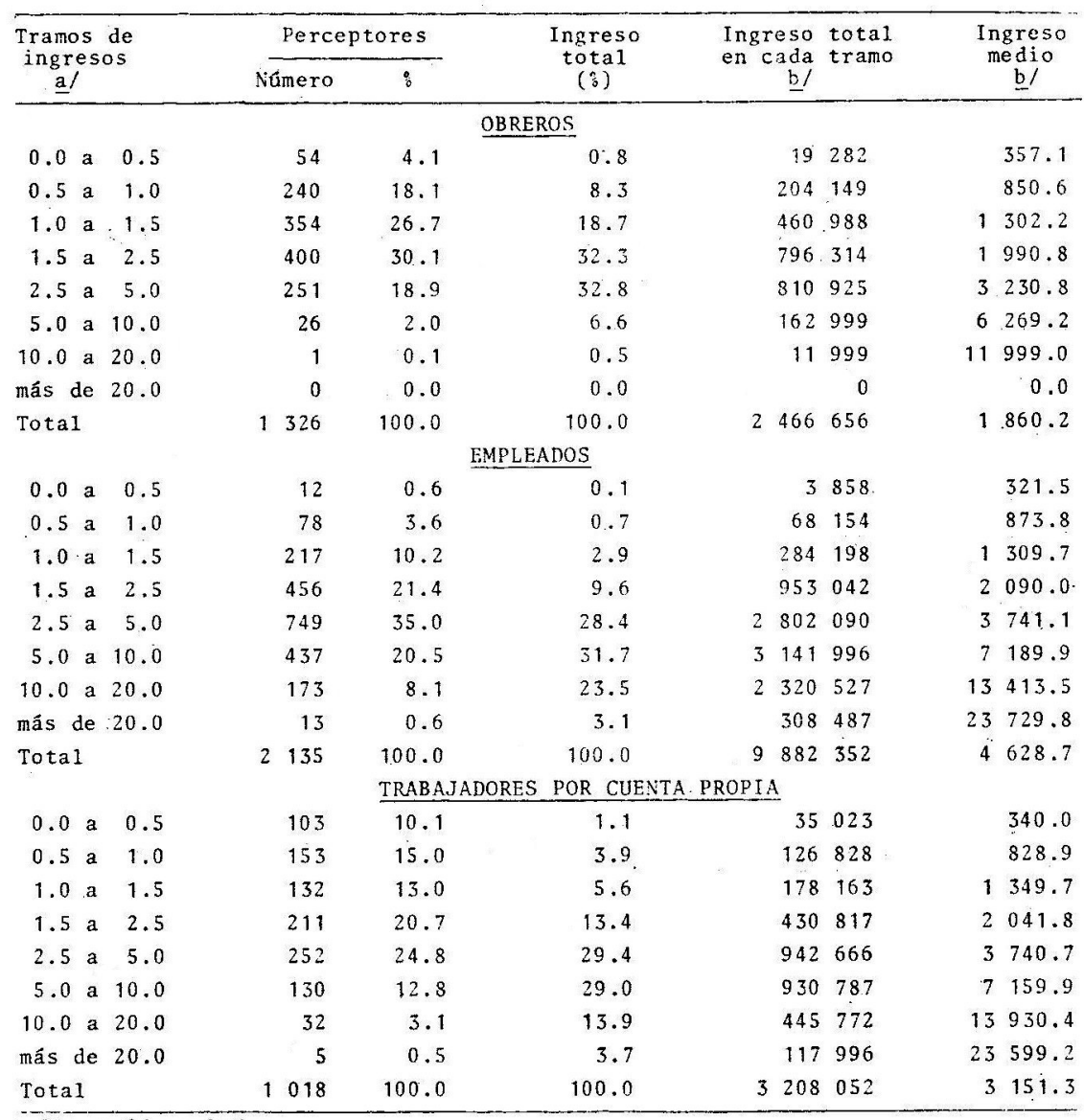

a En sueldos vitales.

b En escudos. 


\section{Cuadro 9}

Gran Santiago: Distribución DEl ingreso PERsonal por tramos, EN SUELDOS VITALES, EN CADA CATEGORÍA OCUPACIONAL, 1972

\begin{tabular}{|c|c|c|c|c|c|c|c|}
\hline \multirow{2}{*}{$\begin{array}{l}\text { Tramos de } \\
\text { ingresos } \\
\text { a/ }\end{array}$} & \multicolumn{2}{|c|}{ Perceptores } & \multirow{2}{*}{$\begin{array}{c}\text { Ingreso } \\
\text { total } \\
\left(\frac{0}{0}\right)\end{array}$} & \multirow{2}{*}{$\begin{array}{l}\text { Ingreso } \\
\text { en cada } \\
\text { b/ }\end{array}$} & \multirow{2}{*}{$\begin{array}{l}\text { total } \\
\text { tramo }\end{array}$} & \multirow{2}{*}{\multicolumn{2}{|c|}{$\begin{array}{c}\text { Ingreso } \\
\text { medio } \\
\text { b/ }\end{array}$}} \\
\hline & Número & $\vdots$ & & & & & \\
\hline \multicolumn{8}{|c|}{ EMPLEADORES } \\
\hline 0.0 a 0.5 & 1 & 0.8 & 0.1 & & 399 & & 399.0 \\
\hline 0.5 a 1.0 & 1 & 0.8 & 0.1 & & 999 & & 999.0 \\
\hline $1.0 \mathrm{a}, 1.5$ & 0 & 0.0 & 0.0 & & $Q$ & & 0.0 \\
\hline $1.5 \mathrm{a} \quad 2.5$ & 4 & 3.2 & 0.6 & 8 & 396 & & 099.0 \\
\hline 2.5 a 5.0 & 23 & 17.4 & 6.9 & 90 & 781 & & 947.0 \\
\hline 5.0 a 10.0 & 53 & 40.0 & 31.2 & 409 & 450 & 7 & 725.5 \\
\hline 10.0 a 20.0 & 41 & 31.0 & 43.1 & 565 & 361 & 13 & 789.3 \\
\hline más de 20.0 & 9 & 6.8 & 18.0 & 235 & 491 & 26 & 165.7 \\
\hline Total & 132 & 100.0 & 100.0 & 1310 & 877 & 9 & 930.9 \\
\hline \multicolumn{8}{|c|}{ EUERZAS ARMADAS } \\
\hline $0.0 \mathrm{a}$ & 0 & 0.0 & 0.0 & & 0 & & 0.0 \\
\hline $0.5 \mathrm{a} \quad 1.0$ & 0 . & 0.0 & 0.0 & & 0 & & 0.0 \\
\hline 1.0 a 1.5 & 2 & 4.7 & 1.2 & 2 & 598 & 1 & 299.0 \\
\hline $1.5 a 2.5$ & 4 & 9.3 & 3.6 & 7 & 846 & & 961.5 \\
\hline 2.5 a 5.0 & 20 & 46.4 & 30.9 & 67 & 261 & 3 & 363.1 \\
\hline 5.0 a 10.0 & 14 & 32.6 & 48.9 & 106 & 216 & 7 & 586.9 \\
\hline 10.0 a 20.0 & 3 & 7.0 & 15.4 & 33 & 497 & 11 & 165.7 \\
\hline más de 20.0 & 0 & 0.0 & 0.0 & & 0 & & 0.0 \\
\hline Total & 43 & 100.0 & 100.0 & 217 & 418 & 5 & 056.2 \\
\hline \multicolumn{8}{|c|}{ EMPLEADOS DOMESTICOS } \\
\hline 0.0 a 0.5 & 13 & 3.1 & 0.8 & 5 & 107 & & 392.9 \\
\hline $0.5 \mathrm{a} \quad 1.0$ & 101 & 24.1 & 13.9 & 84 & 810 & & 839.7 \\
\hline $1.0 \mathrm{a} \quad 1.5$ & 150 & 35.8 & 30.7 & 187 & 444 & 1 & 249.6 \\
\hline 1.5 a 2.5 & 134 & 32.0 & 41.7 & 253 & 846 & 1 & 894.4 \\
\hline 2.5 a 5.0 & 18 & 4.3 & 9.4 & 57 & 152 & 3 & 175.1 \\
\hline 5.0 a 10.0 & 3 & 0.7 & 3.5 & 21 & 547 & 7 & 182.3 \\
\hline $10.0 \mathrm{a} 20.0$ & 0 & 0.0 & 0.0 & & 0 & & 0.0 \\
\hline más de 20.0 & 0 & 0.0 & 0.0 & & 0 & & 0.0 \\
\hline Total & 419 & 100.0 & 100.0 & 609 & 906 & 1 & 455.6 \\
\hline
\end{tabular}

a En sueldos vitales.

b En escudos. 


\section{ANEXo 2}

\section{CALCULO DE LA INTER E INTRAVARIANZA}

1. Procedimiento de cálculo de la intervarianza para los años 1970, 1971 y 1972.

De acuerdo con la información del cuadro 1 del Anexo 1 se ha procedido a calcular la intervarianza para 1970 (véase el cuadro A2-1).

Este procedimiento de cálculo de la intervarianza se ha aplicado a los cuadros 4 y 7 del Anexo 1. En los cuadros A2-2 y A2-3 se presentan los resultados resumidos correspondientes a los años 1971 y 1972, respectivamente.

\section{Cuadro A2-1}

\begin{tabular}{|c|c|c|c|c|c|}
\hline Categoría de ocupación & $\begin{array}{c}\text { Inoreso } \\
\text { medio } \\
\text { (escudos) } \\
(\bar{Y} g)\end{array}$ & $\begin{array}{l}\text { Nímero } \\
\text { de } \\
\text { perceptores } \\
\text { (ng) }\end{array}$ & $\begin{array}{l}\text { Pronorción } \\
\text { de } \\
\text { perceptores } \\
\text { (ig) }\end{array}$ & $\left(\bar{Y}_{g}-\bar{Y}\right)^{2} \mathrm{hg}$ & $(\overline{\mathrm{Y}} \mathrm{g}-\overline{\mathrm{Y}})^{2} \mathrm{hg} / \overline{\mathrm{Y}}^{\mathrm{Z}}$ \\
\hline Obreros & 809.3 & 1533 & 0.311 & 249564.3. & 0.086 \\
\hline Empleados & 2381.3 & 1790 & 0.362 & 165523.2 & 0.057 \\
\hline $\begin{array}{l}\text { Trabajadores por cuenta } \\
\text { propia }\end{array}$ & 1536.9 & 951 & 0.193 & 5460.2 & 0.002 \\
\hline Empleadores & 8822.6 & 115 & 0.023 & 1165152.5 & 0.400 \\
\hline Fuerzas amadas & 1923.4 & 43 & 0.009 & 428.9 & 0.000 \\
\hline Empleados domesticos & 698.6 & 502 & 0.102 & 103330.3 & 0.035 \\
\hline
\end{tabular}

Cuadro A2-2

\begin{tabular}{|c|c|c|}
\hline $\begin{array}{l}\text { Categoría de } \\
\text { ocupación }\end{array}$ & $\left(\bar{Y}_{g}-\bar{\gamma}\right)^{2} h g$ & $\left(\bar{Y}_{g}-\bar{Y}\right)^{2} h g / \bar{Y}^{2}$ \\
\hline $\begin{array}{l}\text { Obreros } \\
\text { Empleados } \\
\text { Trabajadores por cuenta propia } \\
\text { Empleadores } \\
\text { Fuerzas armadas } \\
\text { Empleados domesticos }\end{array}$ & $\begin{array}{rl}406 & 544.0 \\
446 & 249.3 \\
37 & 497.8 \\
740 & 683.4 \\
+1 & 343.8 \\
224 & 535.6\end{array}$ & $\begin{array}{l}0.068 \\
0.074 \\
0.006 \\
0.123 \\
0.001 \\
0.037\end{array}$ \\
\hline
\end{tabular}

Cuadro A2-3

\begin{tabular}{lrr}
\hline $\begin{array}{l}\text { Categoria de } \\
\text { ocupacion }\end{array}$ & $\left(\bar{Y}_{\mathrm{g}}-\overline{\mathrm{Y}}\right)^{2} \mathrm{hg}$ & $\left(\overline{\mathrm{Y}}_{\mathrm{g}}-\overline{\mathrm{Y}}\right)^{2} \mathrm{hg} / \overline{\mathrm{Y}}^{2}$ \\
\hline Obreros & 691665.2 & 0.057 \\
Empleados & 547707.7 & 0.045 \\
Trabajadores por cuenta propia & 22800.3 & 0.002 \\
Empleadores & 1079251.4 & 0.088 \\
Fuerzas armadas & 19671.5 & 0.002 \\
Empleados domesticos & 342877.7 & 0.028 \\
\hline & $\mathrm{S}_{\mathrm{e}, 72}^{2}=2703973.5$ & $\mathrm{~S}_{\mathrm{e}, 72}^{2} / \bar{Y}_{72}^{2}=0.222$
\end{tabular}




\section{Procedimiento de cálculo de la intravarianza}

El cálculo de la intravarianza se realiza con la distribución por tramos de ingresos (en sueldos vitales) para cada una de las seis categorías ocupacionales.

Así por ejemplo, la intravarianza de los obreros para 1970 se calcula con la información correspondiente a obreros del cuadro 2 del Anexo 1 (los resultados aparecen en el cuadro A2-4).

De la misma manera se calculan las intravarianzas de todas las categorías ocupacionales para 1970, utilizando la información de los cuadros 2 y 3 del Anexo 1 (véanse los resultados en el cuadro A2-5).

Para obtener la intravarianza total del año 1970 se pondera cada uno de estos valores por la importancia relativa de cada categoría ocupacional dentro del total. El resultado de esta operación se divide luego por el cuadrado de la media del año (corrección por el efecto de escala) (véase el cuadro A2-6).

Por último, al repetir este procedimiento para 1971 y 1972, se obtienen los resultados que se incluyen en los cuadros A2-7 y A2-8, respectivamente.

\section{Cuadro A2-4}

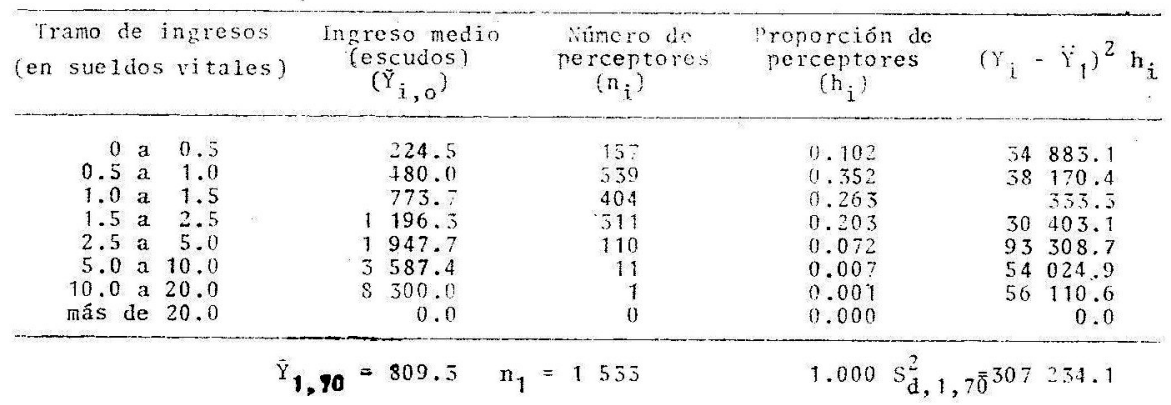

Cuadro A2-5

\begin{tabular}{llr} 
Empleados & $\mathrm{s}_{\mathrm{d}, 2,70}^{2}$ & 4043045.6 \\
Trabajadores por cuenta propia & $\mathrm{s}_{\mathrm{d}, 3,70}^{2}$ & 2636032.0 \\
Empleadores & $\mathrm{s}_{\mathrm{d}, 4,70}^{2}$ & 37891138.0 \\
Fuezzas armadas & $\mathrm{s}_{\mathrm{d}, 5,70}^{2}=$ & 1432078.4 \\
& $\mathrm{~s}_{\mathrm{d}, 6,70}^{2}=$ & 233484.8 \\
\hline
\end{tabular}




\section{Cuadro A2-6}

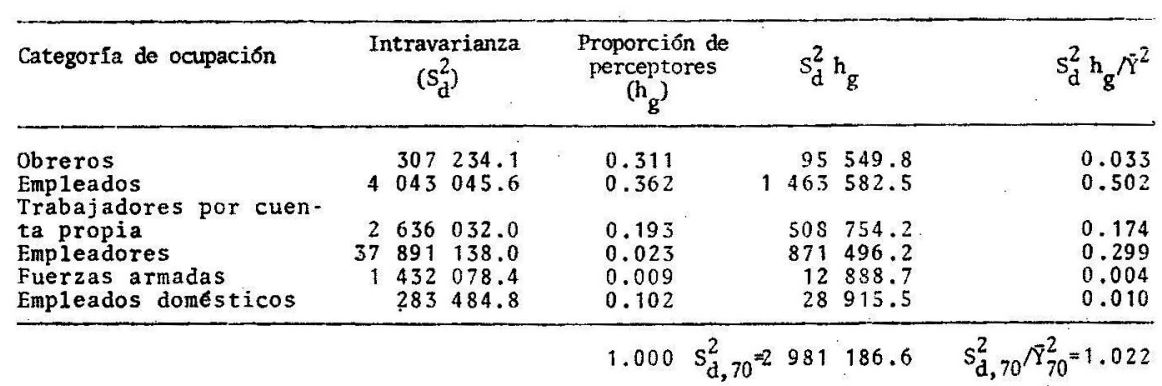

Cuadro A2-7

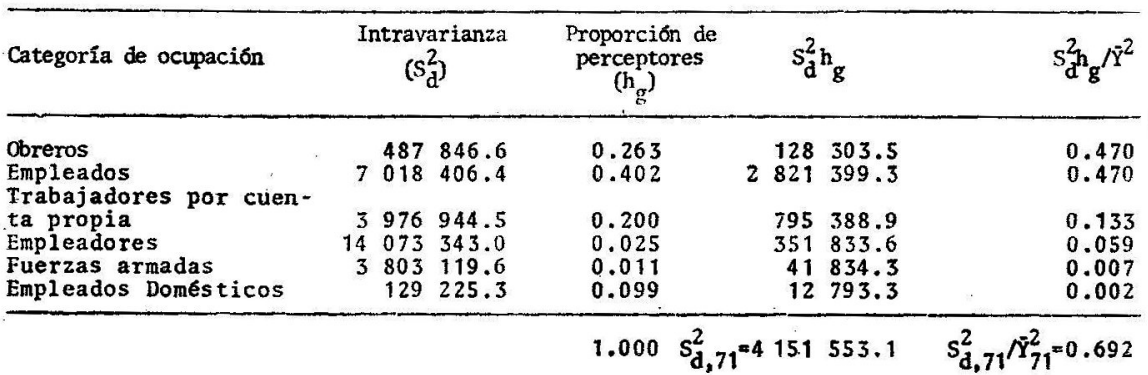

Cuadro A2-8

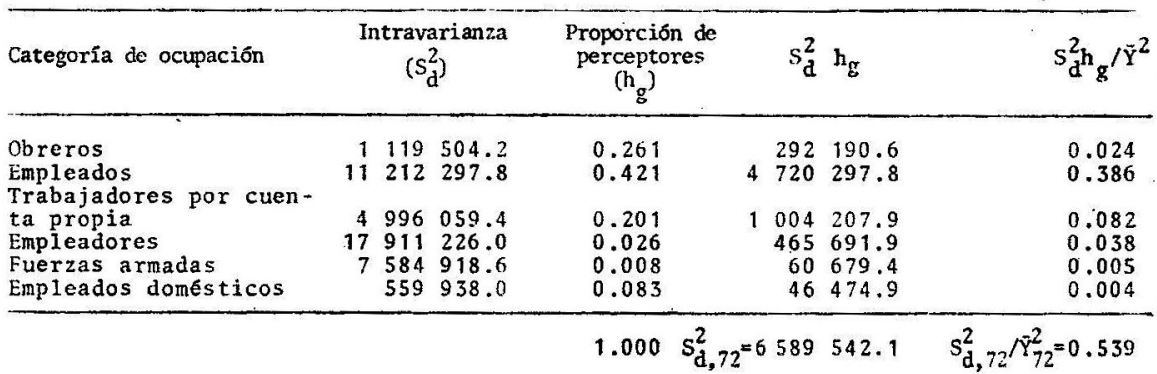

